\title{
Dissection of two drug-targeted regions of Hepatitis C virus subtype 4a infecting Egyptian patients
}

\author{
Radwa R. El-Tahan ${ }^{1}$ (D) Ahmed M. Ghoneim ${ }^{1}$ (1) $\cdot$ Hosam Zaghloul ${ }^{2}$
}

Received: 21 December 2019 / Accepted: 16 June 2020 / Published online: 22 June 2020

○) Springer Science+Business Media, LLC, part of Springer Nature 2020

\begin{abstract}
Recently, treatment of HCV infection has been improved after the development of direct acting antivirals (DAAs) which target different viral proteins (NS3-4A, NS5A and NS5B). The activity and effectiveness of these DAAs are affected by the presence of resistance associated substitutions (RASs). This study aimed to characterize HCV genotypes circulating among Egyptian HCV patients, to dissect the full sequences of HCV NS3-4A and NS5B regions, and to characterize RASs associated with NS3-4A and NS5B inhibitors in HCV treatment-naïve patients. Genotyping of $80 \mathrm{HCV}$ samples from treatmentnaïve patients was done using restriction fragment length polymorphism and phylogenetic analysis based on some full NS5B sequences. Results showed the prevalence of HCV subtype 4a. Twenty four new full sequences of NS3-4A and NS5B regions of subtype 4a were deposited in the GenBank database. In general, the substitutions associated with NS3-4A-targeting drugs were absent predicting possible responsiveness of Egyptian HCV patients to these drugs. In addition, the absence of amino acid substitutions associated with resistance to Sofosbuvir may predict good response to treatment with Sofosbuvir. Some amino acid substitutions associated with resistance to different classes of non-nucleoside inhibitors were detected. Further investigations on treated Egyptian HCV patients may evaluate the effectiveness of the massively used drugs. Many predicted T-cell-binding epitopes in NS3-4A and NS5B regions were found to be highly conserved in the currently studied isolates; a finding that might be important for HCV vaccine development. We demonstrated potential NS3 epitopes that could be used in engineering $\mathrm{T}$ cells against $\mathrm{HCV}$ epitopes.
\end{abstract}

Keywords HCV · Genotypes · NS3-4A · NS5B · Antiviral drugs

Edited by Wolfram H. Gerlich.

Electronic supplementary material The online version of this article (https://doi.org/10.1007/s11262-020-01776-y) contains supplementary material, which is available to authorized users.

Ahmed M. Ghoneim

am_ghoneim@du.edu.eg

Radwa R. El-Tahan

ElTahan.Radwa@yahoo.com

Hosam Zaghloul

Hosam_Z@yahoo.com

1 Zoology Department, Faculty of Science, Damietta University, New Damietta, P.O. 34517, Damietta, Egypt

2 Clinical Pathology Department, Faculty of Medicine, Mansoura University, Mansoura, Egypt

\section{Introduction}

Hepatitis $\mathrm{C}$ virus (HCV) is a global health burden and around 71 million people suffer from chronic hepatitis $\mathrm{C}$ infection [1]. HCV is a significant causative agent for liver cirrhosis, hepatocellular carcinoma, and finally mortality and approximately 400 thousand people die from $\mathrm{HCV}$ each year [1].

HCV belongs to Flaviviridae family and forms its own genus hepacivirus. $\mathrm{HCV}$ is a $9.6 \mathrm{~kb}$ single positive stranded RNA virus. Its open reading frame contains three structural (C, E1, and E2) and seven non-structural (P7, NS2, NS3, NS4A, NS4B, NS5A, and NS5B) proteins. HCV is classified into eight genotypes and more than 80 subtypes [2]. There is $31-33 \%$ nucleotide variance between different HCV genotypes and 20-25\% variance within HCV subtypes of the same genotype [3]. Several HCV subtypes circulate in Egypt with subtype 4a predominating [4, 5] followed by genotype 1 which never exceeds $10 \%[4,6]$. HCV genotyping is 
important in choosing the appropriate direct acting antiviral drugs (DAAs) and the treatment duration [7], and in detecting HCV molecular epidemiology [8].

As a result of Interferon/Ribavirin (INF/RBV) treatment drawbacks which include low sustained virological response, long treatment duration, and severe side effects [9], new antiviral treatment protocols were applied worldwide. DAAs target three important viral regions; NS3-4A protease, NS5A region, and NS5B polymerase [10]. Recent treatment regimens for HCV genotype 4-infected patients are the combinations of Sofosbuvir/Velpatasvir, Glecaprevir/Pibrentasvir, Sofosbuvir/Ledipasvir, and Grazoprevir/Elbasvir [11], and Sofosbuvir/Daclatasvir for 12 weeks $[12,13]$.

Even DAAs have a high virological cure rate, a substantial number of patients reaching $15 \%$ fail to eliminate $\mathrm{HCV}$ infection [14]. Treatment failures have been associated with the presence of resistance-associated substitutions (RASs) in the viral regions (NS3-4A, NS5A, and NS5B) targeted by DAAs [7]. The full NS5B is difficult to be amplified, so it is usually divided into three portions for amplification. In addition, the success rate for the third fragment amplification is low [15]. The failure to amplify the full NS5B has been attributed to its high variability [16]. This study aims to characterize HCV genotypes circulating among Egyptian patients, with a special focus on dissecting the sequences of the full length of NS3-4A and NS5B regions of HCV subtype $4 \mathrm{a}$.

\section{Patients and methods}

\section{Blood sampling}

Blood samples from $80 \mathrm{HCV}$ treatment-naïve patients from Damietta governorate were procured in EDTA-rinsed vacuotainer tubes. The 80 patients included 55 males and 25 females and their ages ranged from 25 to 66 years. All patients were living in the same governorate but in different localities. Our institution board has approved this study (No. 5/2/2/1) and each patient has given an informed consent sample collection.

\section{Viral RNA extraction}

Viral RNA from all blood samples was extracted by GeneJET Viral DNA/RNA Purification Kit (Thermo Scientific, USA) following the standard instructions.

\section{Polymerase chain reaction (PCR)}

Viral RNA of all HCV samples was converted into cDNA using RevertAid ${ }^{\mathrm{TM}}$ First Strand cDNA Synthesis Kit (Thermo Scientific, USA) following the standard instructions. The primers used to amplify $302 \mathrm{bp}$-fragment of the 5' UTR, the full length of NS3-4A (2055 bp), and full length of NS5B (1773 bp) are listed in Table 1. PCR reactions included $25 \mu \mathrm{l}$ of Maxima Hot Start PCR master mix kit (Thermo Scientific, USA) or, in some cases, $25 \mu \mathrm{l}$ of Phusion Hot Start II high-fidelity DNA polymerase Thermo Scientific, USA), $2 \mu \mathrm{l}$ of the forward primer and $2 \mu \mathrm{l}$ of the reverse primer, $1 \mu \mathrm{l}$ of $10 \mathrm{mM}$ dNTPs (in case of using Phusion enzyme), and $4 \mu \mathrm{l}$ of each cDNA sample and the reaction was completed to a final volume of $50 \mu \mathrm{l}$ with water. Reactions were loaded into a thermal cycler (Multigene, Labnet, USA) under the following conditions: for Maxima Hot Start PCR master mix kit, an initial enzyme activation for 4 min at $95{ }^{\circ} \mathrm{C}$ was followed by 40 cycles of $30 \mathrm{~s}$ at $95^{\circ} \mathrm{C}$ for denaturation, $30 \mathrm{~s}$ at a selected annealing temperature and 1-2 min at $72{ }^{\circ} \mathrm{C}$ for extension, while for Phusion Hot Start II high-fidelity DNA polymerase, an initial enzyme activation for $30 \mathrm{~s}$ at $98^{\circ} \mathrm{C}$ was followed by 40 cycles of $10 \mathrm{~s}$ at $98{ }^{\circ} \mathrm{C}$ for denaturation, $30 \mathrm{~s}$ at a the annealing temperature. Cycle extension for $2-3 \mathrm{~min}$ at $72^{\circ} \mathrm{C}$ and a final extension for $10 \mathrm{~min}$ at $72{ }^{\circ} \mathrm{C}$ were applied for both enzymes.

\section{Restriction fragment length polymorphism (RFLP)}

Two combinations of restriction endonucleases; MvaI/HinfI and RsaI/HaeIII, were used to digest 5'UTR PCR products. Digested products were run side by side with undigested PCR products in $2.5 \%$ agarose gels for $40 \mathrm{~min}$ in TBE buffer (Thermo Scientific, USA) buffer, stained with Ethidium Bromide and visualized in Photo Doc-IT Imaging system (UVP, USA).

\section{DNA sequencing}

PCR products of the 5'UTR, NS3-4A, and NS5B regions were purified using GeneJET Gel extraction kit (Thermo Scientific, USA) and sequenced using the standard Sanger method at Macrogen sequencing services (South Korea). Virus genotypes were determined based on the sequence of $\mathrm{HCV}$ prototypes deposited in the GenBank database, followed by additional phylogenetic analysis.

\section{Phylogenetic analysis}

Neighbor-Joining method was used to construct the phylogenetic tree by MEGA7 software. Kimura 2-parameter method with discrete gamma distribution was used to calculate the evolutionary distances and 1000 replicates were used to determine the bootstrap values. Around 80 sequences of other HCV subtype 4 isolates were obtained from $\mathrm{HCV}$ database and the GenBank database and used to make the phylogenetic tree. 
Table 1 Primers used to amplify and sequence 5'UTR, NS3-4A and NS5B regions of HCV

\begin{tabular}{|c|c|c|c|c|}
\hline Position & Primer* & Sequence $\left(5^{\prime}-3^{\prime}\right)$ & Reference & Purpose \\
\hline $47-71$ & 5'UTR_F & GTGAGGAACTACTGTCTTCACGCAG & {$[6]$} & 5'UTR amplification and sequencing \\
\hline $348-324$ & 5'UTR_R & TGCTCATGGTGCACGGTCTACGAGA & {$[6]$} & 5'UTR amplification and sequencing \\
\hline $3262-3280$ & AMG_F1 & TTGTGTTCACGCCCATGGA & This study & NS3-4A amplification and sequencing \\
\hline $3314-3333$ & AMG_F2 & TGCGTGCGGAGACATCATAA & This study & NS3-4A amplification and sequencing \\
\hline $3335-3354$ & AMG_F3 & GGGATTACCTGTTTCGGCCA & This study & NS3-4A amplification and sequencing \\
\hline $3546-3565$ & AMG_F4 & TTCCTGGGTACTGCGGTYAA & This study & NS3-4A amplification and sequencing \\
\hline $4018-3997$ & AMG_R1 & GCGACCTGRTAGGTCTGRGGCA & This study & NS3-4A amplification and sequencing \\
\hline $4182-4201$ & AMG_F5 & GGWGTCAGRACYATYACCAC & This study & NS3-4A sequencing \\
\hline $5537-5518$ & AMG_R2 & GGCCTTCTGCTTGAACTGCT & This study & NS3-4A amplification and sequencing \\
\hline $5067-5046$ & AMG_R3 & TCTGGGATAGGAAATGACCGTC & This study & NS3-4A amplification and sequencing \\
\hline $5153-5172$ & AMG_F6 & GGACACCATGTGGAAGTGCC & This study & NS3-4A sequencing \\
\hline $5961-5941$ & AMG_R4 & GCATCTCGCCGCTCATGATCT & This study & NS3-4A amplification and sequencing \\
\hline $7596-7615$ & AMG_F7 & TGCTGYTCVATGTCMTAYTC & This study & NS5B amplification and sequencing \\
\hline $7596-7615$ & AMG_F8 & TGCTGTTCVATGTCATAYTC & This study & NS5B sequencing \\
\hline $8256-8276$ & JA231_F & TATGAYACCCGCTGYTTTGAC & [17] & NS5B amplification and sequencing \\
\hline $8616-8636$ & JA231_R & CCTGGTCATAGCCTCCGTGAA & [17] & NS5B sequencing \\
\hline $8814-8834$ & AMG_F9 & GTCAATTCCTGGTTGGGAAAC & This study & NS5B sequencing \\
\hline $8833-8814$ & AMG_R5 & TTTCCCAACCAGGAATTGAC & This study & NS5B amplification and sequencing \\
\hline 9395-9376 & AMG_R6 & GTCGGAGTGTTAAGCTGCCT & This study & NS5B amplification and sequencing \\
\hline $9372-9353$ & AMG_R7 & CGAGCAGGCAGCAGRAAGATG & This study & NS5B amplification and sequencing \\
\hline
\end{tabular}

*"F" refers to forward primers while " $\mathrm{R}$ " refers to reverse primers

\section{Results}

\section{HCV genotyping by RFLP}

Viral cDNA produced from $80 \mathrm{HCV}$-infected patients was used to amplify 302 bp-fragment of HCV 5'UTR. The 80 5'UTR amplicons were double digested by two pairs of enzymes; MvaI/HinfI and RasI/HaeIII. Most of the restriction digestion patterns were as described in our previous study [4] (Fig. 1a) and indicated the presence of two different HCV genotypes; most probably genotype 1 and genotype 4. Some samples, however, showed irregular digestion patterns. For example, sample 090 had an irregular MvaI/HinfI digestion pattern (Fig. 1a, II); two clear $\sim 177$ bp $\sim 129$ bp bands and two small close bands ( $\sim 56$ and $\sim 69$ bp). Collectively, the regular and irregular digestion patterns showed the presence of 2 different HCV genotypes; most probably genotype 1 (represented by sample 069 in Fig. 1a, I \& III and sample 090 in Fig. 1a, II) and genotype 4 (represented by sample 068 in Fig. 1a, I \& III).

\section{Amplification of the full length of NS5B region}

Out of the $80 \mathrm{HCV}$ samples, twelve samples were used for sequencing the full length of the NS5B region. The twelve selected samples were from non-related patients living in Damietta Governorate but in different localities. They were 8 males and 4 females and their age ranged from 40-55 years. Five patients ( 2 males and 3 females) had a low viral load (77.500 to $672.000 \mathrm{IU} / 1)$ and seven patients (6 males and 1 female) had a high viral load (1.080.000 to 5.000.000 IU/1).

To amplify the full NS5B gene $(1773 \mathrm{bp})$, three pairs of primers were used in separate PCR reactions. The primer pair AMG_F7/AMG_R5 was used to amplify the 5' part (1238 bp fragment) of the NS5B gene (Fig. 1b, I). The primer pair JA231_F/AMG_R7 was used to amplify the 3' part (1117 bp fragment) of the NS5B gene (Fig. 1b, II). In some cases, the primer pair JA231_F_AMG_R6 was alternatively used to amplify the $3^{\prime}$ part (1140 bp fragment) of the NS5B gene (Fig. 1b, III). Sequences of the full length of NS5B region (1773 bp) of the twelve sequenced samples (083, 087, 093, 094, 095, 103, 105, 108, 113, 115, 122, and 126) were aligned to the NCBI database and found to represent new isolates. These sequences were submitted to the GenBank database under the accession numbers MG602797-MG602808.

\section{Amplification of the full length of NS3-4A region}

The same twelve HCV isolates sequenced for their NS5B gene were used for sequencing the full length of NS3-4A genes. To amplify the full NS3-4A region (2055 bp), five pairs of primers were used in separate PCR reactions. The primer pair AMG_F1_AMG_R1 was used to amplify the 5' 
A $\quad M v a \mathrm{I} /$ Hinfl

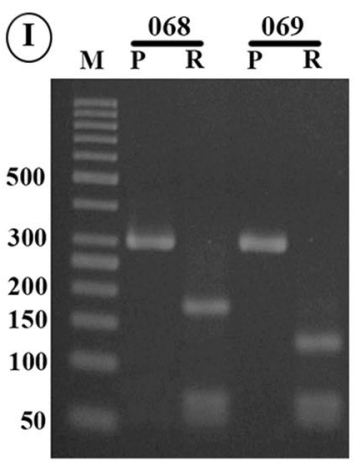

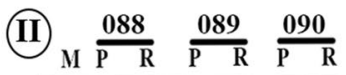

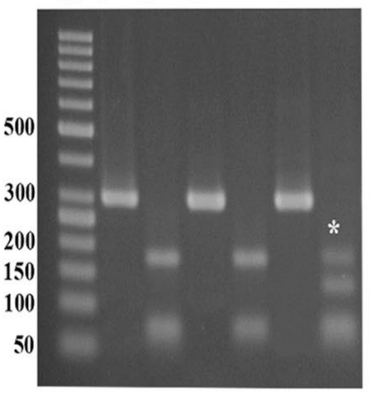

RsaI/HaeIII

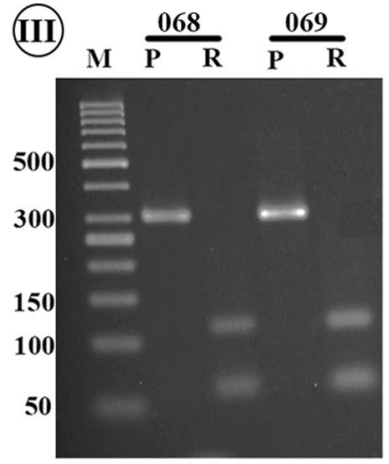

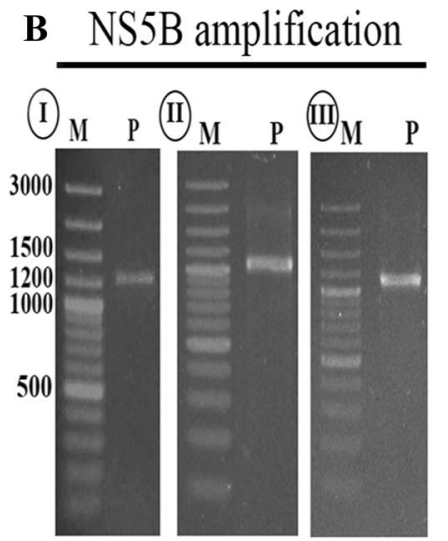

C

NS3-4A amplification

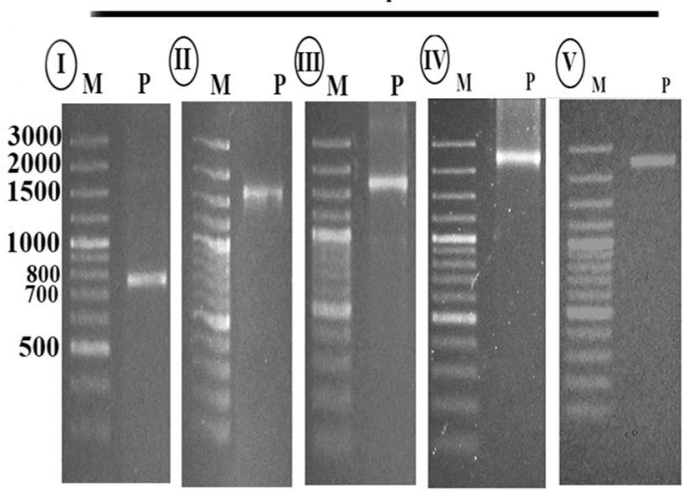

Fig. 1 a RFLP of 5'UTR for representative HCV samples. I: double digestion with MvaI/HinfI (regular pattern). II: double digestion with MvaI/Hinfl, lane designated "*” represents irregular MvaI/HinfI double digestion pattern. III: double digestion with RsaI/HaeIII. Wells designated " $\mathrm{P}$ " show undigested PCR products and wells designated " $R$ " show digested products. " $M$ " designates the well containing a 50 bp DNA ladder. Sample 069 and sample 090 represent type 1 cleavage pattern (a, I, II \& III) and sample 068 represents type 4 cleavage pattern (a, I \& III). b Amplification of the full length of NS5B region. I: The amplification product of the $5^{\prime}$ part of the NS5B gene (expected size $1238 \mathrm{bp}$ ). II: The amplification product of the $3^{\prime}$

part (757 bp fragment) of the NS3-4A region (Fig. 1c, I). In some cases, the primer pairs AMG_F2_AMG_R3 or AMG_ F3_AMG_R3 were used to amplify 1754 bp or 1733 bp, respectively, from the $5^{\prime}$ part of the NS3-4A region (Fig. 1c, II \& III). The remaining 3' part of the NS3-4A region was covered by amplifying $2416 \mathrm{bp}$ fragment by the primer pair AMG_F4_AMG_R3 (Fig. 1c, IV). This fragment overlaps a major part of the amplified 5' part of the NS3-4A region. In some other cases, the full NS3-4A region (in a 2648 bp fragment) was amplified in a single reaction by the primer pair AMG_F2_AMG_R4 (Fig. 1c, V). The twelve isolates were sequenced for their full length of NS3-4A region (2055 bp) and their sequences were found to be new and thus submitted to the GenBank database under the accession numbers MK210544-MK210555. part of the NS5B gene (expected size $1117 \mathrm{bp}$ ). III: The amplification product of the 3' part of the NS5B gene (expected size $1140 \mathrm{bp}$ ) using a different primer pair. c Amplification of the full length of NS3-4A region. I: The amplification product of the $5^{\prime}$ part of the NS3-4A gene (expected size $757 \mathrm{bp}$ ). II, III: The amplification products of the $5^{\prime}$ part of the NS3-4A gene (expected size $1754 \mathrm{bp}$ in B and $1733 \mathrm{bp}$ in c) using different primer pairs. IV: The amplification product of the $3^{\prime}$ part of the NS3-4A gene (expected size $2416 \mathrm{bp}$ ). V: The amplification product of the full length of the NS3-4A gene (expected size $2648 \mathrm{bp}$ )

\section{Phylogenetic analysis}

NS5B sequences of the twelve isolates under study were subjected to phylogenetic analysis. The $1773 \mathrm{bp}$ composing the full length of NS5B of these isolates were aligned with 80 HCV subtype 4 sequences, comprising reference sequences plus all the sequences from Egyptian HCV isolates, and a Neighbor-Joining phylogenetic tree was constructed (Fig. 2). The twelve studied isolates clustered with HCV subtype 4a from Egypt and from USA with high bootstrap values.

\section{Variability of the full length of NS5B region}

Compared to the reference subtype $4 \mathrm{a}(\mathrm{Y} 11604)$ and the reference prototype 1a (NC_004102), analysis of the amino 
Fig. 2 Neighbor-Joining phylogenetic tree of the full length sequence of NS5B (positions 7602-9374) of several HCV genotype 4a representatives. Bootstrap values based on 1000 replicates are shown next to the branches; bootstrap values more than $50 \%$ only are shown. Sequences are labeled to the right of each branch in the order: Gene Bank accession number, isolate name, genotype/ subtype and country. Sequences from reference genomes are referred to with "Ref" after the subtype name. Sequences of the current study are underlined and followed by "current study"

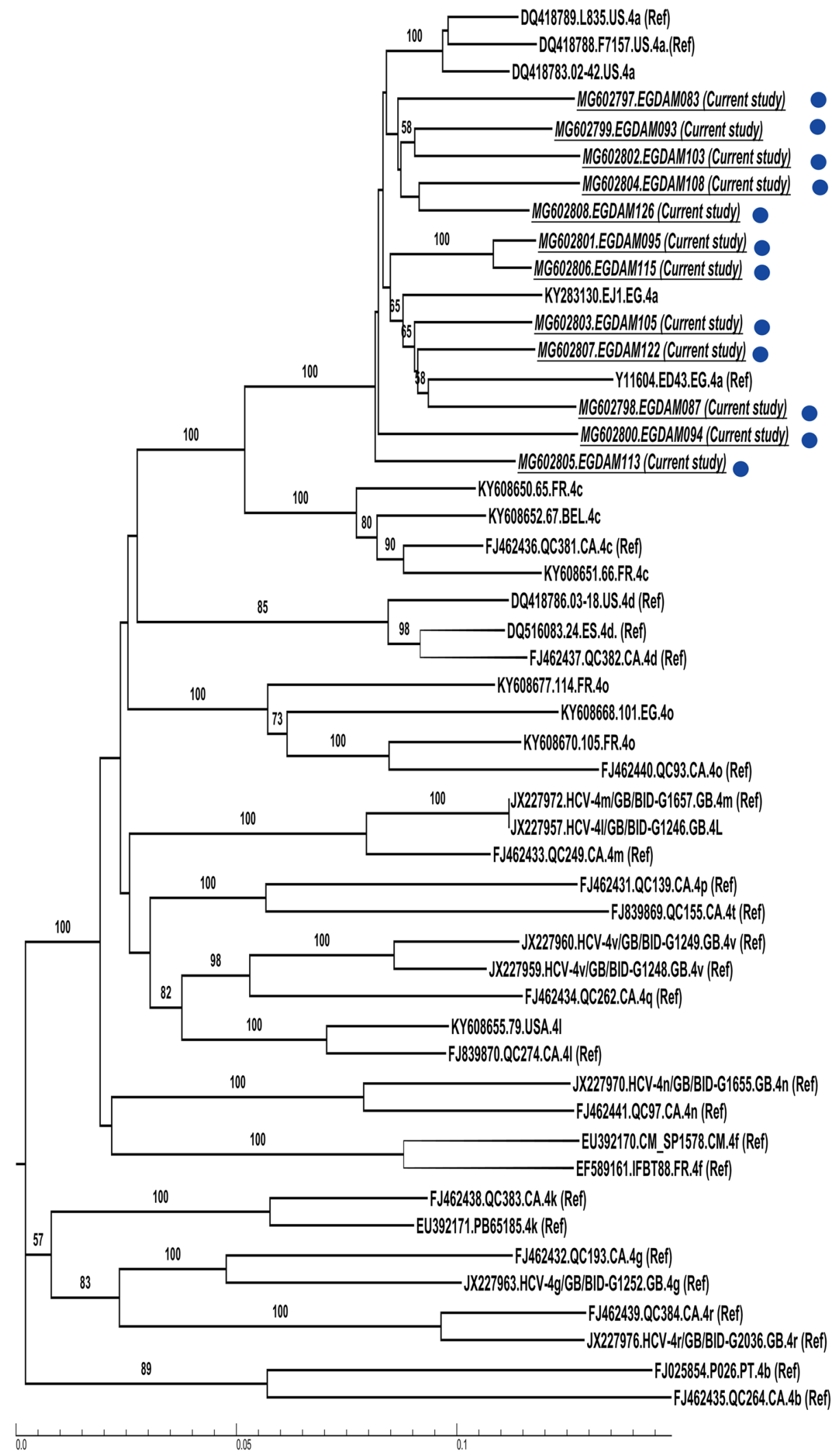


Table 2 Amino acid variance in NS5B region of $12 \mathrm{HCV}$ isolates

\begin{tabular}{|c|c|c|c|c|c|c|c|c|c|c|c|c|c|c|}
\hline \multicolumn{2}{|c|}{$\begin{array}{l}\text { 1a. H77 } \\
\text { NC_004102 }\end{array}$} & $\begin{array}{l}4 a \\
\text { ED43 } \\
Y 11604\end{array}$ & $\begin{array}{l}\text { EG } \\
\text { DAM } \\
083\end{array}$ & $\begin{array}{l}\text { EG } \\
\text { DAM } \\
087\end{array}$ & $\begin{array}{l}\text { EG } \\
\text { DAM } \\
093\end{array}$ & $\begin{array}{l}\text { EG } \\
\text { DAM } \\
094\end{array}$ & $\begin{array}{l}\text { EG } \\
\text { DAM } \\
095\end{array}$ & $\begin{array}{l}\text { EG } \\
\text { DAM } \\
103\end{array}$ & $\begin{array}{l}\text { EG } \\
\text { DAM } \\
105\end{array}$ & $\begin{array}{l}\text { EG } \\
\text { DAM } \\
108\end{array}$ & $\begin{array}{l}\text { EG } \\
\text { DAM } \\
113\end{array}$ & $\begin{array}{l}\text { EG } \\
\text { DAM } \\
115\end{array}$ & $\begin{array}{l}\text { EG } \\
\text { DAM } \\
122\end{array}$ & $\begin{array}{l}\text { EG } \\
\text { DAM } \\
126\end{array}$ \\
\hline 10 & $\mathrm{~L}$ & $\mathrm{~L}$ & $\mathrm{~L}$ & $\mathrm{~F}$ & $\mathrm{~L}$ & $\mathrm{~L}$ & $\mathrm{~L}$ & $\mathrm{~L}$ & $\mathrm{~L}$ & $\mathrm{~L}$ & $\mathrm{~L}$ & $\mathrm{~L}$ & $\mathrm{~L}$ & $\mathrm{~L}$ \\
\hline 18 & E & $\mathrm{E}$ & $\mathrm{E}$ & $\mathrm{G}$ & $\mathrm{G}$ & $\mathrm{E}$ & $\mathrm{E}$ & $\mathrm{G}$ & $\mathrm{E}$ & $\mathrm{E}$ & $\mathrm{E}$ & $\mathrm{G}$ & $\mathrm{E}$ & $\mathrm{E}$ \\
\hline 19 & $\mathrm{Q}$ & $\mathrm{S}$ & $\mathrm{S}$ & $S$ & $S$ & $\mathrm{~S}$ & $\mathrm{~S}$ & $S$ & $S$ & $S$ & $\mathrm{~S}$ & $\mathrm{~S}$ & $\mathrm{~S}$ & $S$ \\
\hline 24 & $\mathrm{~N}$ & $\mathrm{~S}$ & $\mathrm{~S}$ & $\mathrm{~S}$ & $S$ & $\mathrm{~S}$ & $\mathrm{~S}$ & $\mathrm{~S}$ & $\mathrm{~S}$ & $\mathrm{~S}$ & $\mathrm{~S}$ & $\mathrm{~S}$ & $\mathrm{~S}$ & $S$ \\
\hline 25 & A & $\mathrm{P}$ & $\mathrm{P}$ & $\mathrm{P}$ & $\mathrm{P}$ & $\mathrm{P}$ & $\mathrm{P}$ & $\mathrm{P}$ & $\mathrm{P}$ & $\mathrm{P}$ & $\mathrm{P}$ & $\mathrm{P}$ & $\mathrm{P}$ & $\mathrm{P}$ \\
\hline 36 & $\mathrm{~L}$ & $\mathrm{M}$ & $\mathrm{M}$ & $\mathrm{M}$ & $\mathrm{M}$ & $\mathrm{M}$ & $\mathrm{M}$ & $\mathrm{M}$ & $\mathrm{M}$ & $\mathrm{M}$ & $\mathrm{M}$ & $\mathrm{M}$ & $\mathrm{M}$ & $\mathrm{M}$ \\
\hline 39 & $\mathrm{~S}$ & A & A & A & A & A & A & A & A & A & $\mathrm{A}$ & $\mathrm{A}$ & $\mathrm{A}$ & A \\
\hline 42 & $\mathrm{~S}$ & $\mathrm{~T}$ & $\mathrm{~T}$ & $\mathrm{~T}$ & $\mathrm{~T}$ & $\mathrm{~T}$ & $\mathrm{~T}$ & $\mathrm{~T}$ & $\mathrm{~T}$ & $\mathrm{~T}$ & $\mathrm{~T}$ & $\mathrm{~T}$ & $\mathrm{~T}$ & $\mathrm{~T}$ \\
\hline 46 & $\mathrm{C}$ & $\mathrm{V}$ & $\mathrm{V}$ & V & V & $\mathrm{V}$ & $\mathrm{V}$ & $\mathrm{V}$ & V & $\mathrm{V}$ & $\mathrm{V}$ & $\mathrm{V}$ & V & V \\
\hline 47 & $\mathrm{Q}$ & $\mathrm{T}$ & $\mathrm{T}$ & $\mathrm{T}$ & A & $\mathrm{M}$ & $\mathrm{T}$ & $\mathrm{T}$ & $\mathrm{T}$ & $\mathrm{T}$ & $\mathrm{T}$ & $\mathrm{T}$ & $\mathrm{T}$ & A \\
\hline 60 & $\mathrm{~L}$ & V & $\mathrm{L}$ & V & V & V & V & $\mathrm{V}$ & V & V & V & V & $\mathrm{V}$ & $\mathrm{V}$ \\
\hline 62 & $\mathrm{~S}$ & $\mathrm{~S}$ & $\mathrm{D}$ & $\mathrm{N}$ & $\mathrm{N}$ & $\mathrm{Q}$ & $\mathrm{N}$ & $\mathrm{N}$ & $\mathrm{N}$ & $\mathrm{N}$ & $\mathrm{N}$ & $\mathrm{N}$ & $\mathrm{H}$ & $\mathrm{N}$ \\
\hline 63 & $\mathrm{H}$ & $\mathrm{T}$ & $\mathrm{H}$ & $\mathrm{Y}$ & $\mathrm{H}$ & $\mathrm{H}$ & $\mathrm{H}$ & $\mathrm{H}$ & $\mathrm{H}$ & $\mathrm{H}$ & $\mathrm{H}$ & $\mathrm{H}$ & $\mathrm{H}$ & $\mathrm{H}$ \\
\hline 65 & $\mathrm{Q}$ & $\mathrm{N}$ & $\mathrm{N}$ & $\mathrm{N}$ & $\mathrm{N}$ & $\mathrm{N}$ & $\mathrm{T}$ & $\mathrm{N}$ & $\mathrm{N}$ & $\mathrm{N}$ & $\mathrm{N}$ & $\mathrm{T}$ & $\mathrm{N}$ & $\mathrm{N}$ \\
\hline 66 & $\mathrm{D}$ & $\mathrm{E}$ & A & $\mathrm{E}$ & $\mathrm{E}$ & $\mathrm{D}$ & $\mathrm{E}$ & $\mathrm{E}$ & E & $\mathrm{E}$ & $\mathrm{E}$ & $\mathrm{E}$ & $\mathrm{E}$ & $\mathrm{D}$ \\
\hline 71 & $\mathrm{~V}$ & I & I & I & I & I & I & I & I & I & I & I & I & I \\
\hline 74 & A & $\mathrm{R}$ & $\mathrm{R}$ & $\mathrm{R}$ & $\mathrm{R}$ & $\mathrm{R}$ & $\mathrm{R}$ & $\mathrm{R}$ & $\mathrm{R}$ & $\mathrm{R}$ & $\mathrm{R}$ & $\mathrm{R}$ & $\mathrm{R}$ & $\mathrm{R}$ \\
\hline 77 & $\mathrm{~K}$ & $\mathrm{R}$ & $\mathrm{R}$ & $\mathrm{R}$ & $\mathrm{R}$ & $\mathrm{K}$ & $\mathrm{R}$ & $\mathrm{K}$ & $\mathrm{R}$ & $\mathrm{R}$ & $\mathrm{R}$ & $\mathrm{R}$ & $\mathrm{R}$ & $\mathrm{T}$ \\
\hline 80 & A & $\mathrm{P}$ & A & A & A & A & A & A & A & A & $\mathrm{A}$ & A & A & A \\
\hline 81 & $\mathrm{~N}$ & $\mathrm{R}$ & $\mathrm{R}$ & $\mathrm{R}$ & $\mathrm{R}$ & $\mathrm{R}$ & $\mathrm{R}$ & $\mathrm{R}$ & $\mathrm{R}$ & $\mathrm{R}$ & $\mathrm{R}$ & $\mathrm{R}$ & $\mathrm{R}$ & $\mathrm{R}$ \\
\hline 85 & V & $\mathrm{T}$ & $\mathrm{T}$ & $\mathrm{T}$ & $\mathrm{T}$ & $\mathrm{T}$ & $\mathrm{T}$ & $\mathrm{T}$ & $\mathrm{T}$ & $\mathrm{T}$ & $\mathrm{T}$ & $\mathrm{T}$ & $\mathrm{T}$ & $\mathrm{T}$ \\
\hline 90 & $\mathrm{~S}$ & $\mathrm{D}$ & $\mathrm{D}$ & D & $\mathrm{D}$ & $\mathrm{D}$ & $\mathrm{D}$ & $\mathrm{D}$ & D & $\mathrm{D}$ & $\mathrm{D}$ & $\mathrm{D}$ & $\mathrm{N}$ & D \\
\hline 98 & $\mathrm{~K}$ & $\mathrm{R}$ & $\mathrm{K}$ & $\mathrm{R}$ & $\mathrm{R}$ & $\mathrm{R}$ & $\mathrm{K}$ & $\mathrm{R}$ & $\mathrm{R}$ & $\mathrm{R}$ & $\mathrm{R}$ & $\mathrm{K}$ & $\mathrm{R}$ & $\mathrm{R}$ \\
\hline 105 & A & $\mathrm{K}$ & A & A & A & A & A & A & A & A & A & A & A & A \\
\hline 110 & $\mathrm{C}$ & $\mathrm{S}$ & $\mathrm{S}$ & $\mathrm{S}$ & $S$ & $\mathrm{~S}$ & $\mathrm{~N}$ & $\mathrm{~S}$ & $\mathrm{~S}$ & $S$ & $\mathrm{~S}$ & $\mathrm{~N}$ & $\mathrm{~S}$ & $\mathrm{~S}$ \\
\hline 112 & A & $\mathrm{S}$ & $\mathrm{S}$ & $\mathrm{S}$ & $S$ & $\mathrm{~S}$ & $\mathrm{~S}$ & $\mathrm{~S}$ & $S$ & $\mathrm{~S}$ & $\mathrm{~S}$ & $\mathrm{~S}$ & $\mathrm{~S}$ & $S$ \\
\hline 120 & $\mathrm{~N}$ & $\mathrm{~S}$ & $\mathrm{~N}$ & $\mathrm{~N}$ & $\mathrm{~N}$ & $\mathrm{~N}$ & $\mathrm{~N}$ & $\mathrm{~N}$ & $\mathrm{~N}$ & $\mathrm{~N}$ & $\mathrm{~N}$ & $\mathrm{~N}$ & $\mathrm{~N}$ & $\mathrm{~N}$ \\
\hline 124 & $\mathrm{~K}$ & $\mathrm{~K}$ & $\mathrm{E}$ & $\mathrm{E}$ & $\mathrm{E}$ & $\mathrm{K}$ & $\mathrm{E}$ & $\mathrm{E}$ & $\mathrm{E}$ & $\mathrm{E}$ & $\mathrm{E}$ & $\mathrm{E}$ & $\mathrm{E}$ & $\mathrm{E}$ \\
\hline 130 & $\mathrm{~S}$ & $\mathrm{~N}$ & $\mathrm{~N}$ & $\mathrm{~N}$ & $\mathrm{~N}$ & $\mathrm{~N}$ & $\mathrm{~N}$ & $\mathrm{~N}$ & $\mathrm{~N}$ & $\mathrm{~N}$ & $\mathrm{~N}$ & $\mathrm{~N}$ & $\mathrm{~N}$ & $\mathrm{D}$ \\
\hline 135 & $\mathrm{D}$ & $\mathrm{P}$ & $\mathrm{S}$ & $\mathrm{P}$ & $S$ & $\mathrm{P}$ & $\mathrm{P}$ & $\mathrm{P}$ & $\mathrm{P}$ & $\mathrm{S}$ & $\mathrm{P}$ & $\mathrm{P}$ & $\mathrm{Q}$ & $\mathrm{P}$ \\
\hline 146 & $\mathrm{C}$ & A & A & A & V & A & A & A & A & A & A & A & A & A \\
\hline 148 & $\mathrm{Q}$ & $\mathrm{N}$ & $\mathrm{N}$ & $\mathrm{N}$ & $\mathrm{N}$ & $\mathrm{N}$ & $\mathrm{N}$ & $\mathrm{N}$ & $\mathrm{N}$ & $\mathrm{N}$ & $\mathrm{N}$ & $\mathrm{N}$ & $\mathrm{N}$ & $\mathrm{N}$ \\
\hline 149 & $\mathrm{P}$ & $\mathrm{P}$ & $\mathrm{P}$ & $\mathrm{P}$ & $\mathrm{P}$ & $\mathrm{P}$ & $\mathrm{P}$ & Q & $\mathrm{P}$ & $\mathrm{P}$ & $\mathrm{P}$ & $\mathrm{P}$ & $\mathrm{P}$ & $\mathrm{P}$ \\
\hline 150 & $\mathrm{E}$ & A & A & A & A & A & A & $\mathrm{T}$ & A & A & A & A & A & A \\
\hline 156 & $\mathrm{P}$ & $\mathrm{P}$ & A & A & $\mathrm{P}$ & $\mathrm{P}$ & $\mathrm{P}$ & $\mathrm{P}$ & $\mathrm{P}$ & $\mathrm{P}$ & $\mathrm{P}$ & $\mathrm{P}$ & $\mathrm{P}$ & $\mathrm{P}$ \\
\hline 162 & $\mathrm{~F}$ & $\mathrm{Y}$ & $\mathrm{Y}$ & $\mathrm{Y}$ & $\mathrm{Y}$ & $\mathrm{Y}$ & $\mathrm{Y}$ & $\mathrm{Y}$ & $\mathrm{Y}$ & $\mathrm{Y}$ & $\mathrm{Y}$ & $\mathrm{Y}$ & $\mathrm{Y}$ & $\mathrm{Y}$ \\
\hline 167 & $\mathrm{~V}$ & $\mathrm{~S}$ & $\mathrm{~V}$ & $\mathrm{~V}$ & V & $\mathrm{V}$ & $\mathrm{V}$ & $\mathrm{V}$ & $\mathrm{V}$ & $\mathrm{V}$ & $\mathrm{V}$ & $\mathrm{V}$ & V & V \\
\hline 173 & $\mathrm{M}$ & $\mathrm{R}$ & $\mathrm{R}$ & $\mathrm{R}$ & $\mathrm{R}$ & $\mathrm{R}$ & $\mathrm{R}$ & $\mathrm{R}$ & $\mathrm{R}$ & $\mathrm{R}$ & $\mathrm{R}$ & $\mathrm{R}$ & $\mathrm{R}$ & $\mathrm{R}$ \\
\hline 176 & $\mathrm{Y}$ & $\mathrm{H}$ & $\mathrm{R}$ & $\mathrm{H}$ & $\mathrm{Y}$ & $\mathrm{H}$ & $\mathrm{H}$ & $\mathrm{Y}$ & $\mathrm{H}$ & $\mathrm{Y}$ & $\mathrm{H}$ & $\mathrm{H}$ & $\mathrm{H}$ & $\mathrm{Y}$ \\
\hline 178 & $\mathrm{~V}$ & V & I & $\mathrm{V}$ & V & I & V & $\mathrm{V}$ & $\mathrm{V}$ & V & $\mathrm{V}$ & V & $\mathrm{V}$ & V \\
\hline 179 & $\mathrm{~V}$ & I & I & I & I & $\mathrm{V}$ & I & I & I & I & I & I & I & I \\
\hline 180 & $\mathrm{~S}$ & $\mathrm{~K}$ & $\mathrm{~N}$ & $\mathrm{~K}$ & $\mathrm{R}$ & $\mathrm{Q}$ & $\mathrm{K}$ & $\mathrm{K}$ & $\mathrm{K}$ & $\mathrm{N}$ & $\mathrm{N}$ & $\mathrm{K}$ & $\mathrm{K}$ & $S$ \\
\hline 181 & $\mathrm{~K}$ & $\mathrm{~K}$ & $\mathrm{Q}$ & $\mathrm{Q}$ & $\mathrm{Q}$ & $\mathrm{Q}$ & $\mathrm{Q}$ & $\mathrm{Q}$ & Q & $\mathrm{K}$ & $\mathrm{Q}$ & $\mathrm{Q}$ & $\mathrm{Q}$ & Q \\
\hline 182 & $\mathrm{~L}$ & $\mathrm{~T}$ & $\mathrm{~L}$ & $\mathrm{~L}$ & L & $\mathrm{L}$ & $\mathrm{L}$ & $\mathrm{L}$ & $\mathrm{L}$ & $\mathrm{L}$ & $\mathrm{L}$ & $\mathrm{L}$ & $\mathrm{L}$ & $\mathrm{L}$ \\
\hline 183 & $\mathrm{P}$ & A & $\mathrm{P}$ & $\mathrm{P}$ & $\mathrm{P}$ & $\mathrm{P}$ & $\mathrm{P}$ & $\mathrm{P}$ & $\mathrm{P}$ & $\mathrm{P}$ & $\mathrm{P}$ & $\mathrm{P}$ & $\mathrm{P}$ & $\mathrm{P}$ \\
\hline 184 & $\mathrm{~L}$ & $\mathrm{~L}$ & $\mathrm{E}$ & $\mathrm{E}$ & $\mathrm{E}$ & $\mathrm{E}$ & $\mathrm{E}$ & $\mathrm{E}$ & $\mathrm{E}$ & $\mathrm{E}$ & $\mathrm{E}$ & $\mathrm{E}$ & $\mathrm{E}$ & $\mathrm{E}$ \\
\hline 189 & $\mathrm{~S}$ & A & A & A & A & A & A & A & A & A & A & A & A & A \\
\hline 190 & $\mathrm{~S}$ & A & A & A & $S$ & A & A & A & A & A & $\mathrm{A}$ & A & A & A \\
\hline
\end{tabular}


Table 2 (continued)

\begin{tabular}{|c|c|c|c|c|c|c|c|c|c|c|c|c|c|c|}
\hline \multicolumn{2}{|c|}{$\begin{array}{l}\text { 1a. H77 } \\
\text { NC_004102 }\end{array}$} & $\begin{array}{l}4 a \\
\text { ED43 } \\
\text { Y11604 }\end{array}$ & $\begin{array}{l}\text { EG } \\
\text { DAM } \\
083\end{array}$ & $\begin{array}{l}\text { EG } \\
\text { DAM } \\
087\end{array}$ & $\begin{array}{l}\text { EG } \\
\text { DAM } \\
093\end{array}$ & $\begin{array}{l}\text { EG } \\
\text { DAM } \\
094\end{array}$ & $\begin{array}{l}\text { EG } \\
\text { DAM } \\
095\end{array}$ & $\begin{array}{l}\text { EG } \\
\text { DAM } \\
103\end{array}$ & $\begin{array}{l}\text { EG } \\
\text { DAM } \\
105\end{array}$ & $\begin{array}{l}\text { EG } \\
\text { DAM } \\
108\end{array}$ & $\begin{array}{l}\text { EG } \\
\text { DAM } \\
113\end{array}$ & $\begin{array}{l}\text { EG } \\
\text { DAM } \\
115\end{array}$ & $\begin{array}{l}\text { EG } \\
\text { DAM } \\
122\end{array}$ & $\begin{array}{l}\text { EG } \\
\text { DAM } \\
126\end{array}$ \\
\hline 198 & G & A & A & A & A & A & A & A & A & A & A & A & A & A \\
\hline 234 & $\mathrm{R}$ & $\mathrm{R}$ & $\mathrm{R}$ & $\mathrm{R}$ & $\mathrm{R}$ & $\mathrm{R}$ & $\mathrm{R}$ & $\mathrm{R}$ & $\mathrm{R}$ & $\mathrm{K}$ & $\mathrm{R}$ & $\mathrm{R}$ & $\mathrm{R}$ & $\mathrm{R}$ \\
\hline 241 & Q & Q & Q & Q & $\mathrm{L}$ & Q & Q & Q & Q & Q & Q & Q & Q & Q \\
\hline 244 & $\mathrm{D}$ & $\mathrm{D}$ & $\mathrm{D}$ & N/D & $\mathrm{N}$ & $\mathrm{D}$ & $\mathrm{D}$ & $\mathrm{D}$ & $\mathrm{D}$ & $\mathrm{D}$ & $\mathrm{N}$ & $\mathrm{D}$ & $\mathrm{N}$ & $\mathrm{D}$ \\
\hline 246 & D & E & E & E & E & E & E & $\mathrm{E}$ & E & E & E & E & E & E \\
\hline 258 & $\mathrm{E}$ & D & $\mathrm{E}$ & $\mathrm{E}$ & $\mathrm{E}$ & $\mathrm{E}$ & $\mathrm{E}$ & $\mathrm{E}$ & $\mathrm{E}$ & $\mathrm{E}$ & $\mathrm{E}$ & $\mathrm{E}$ & $\mathrm{E}$ & E \\
\hline 282 & $\mathrm{~S}$ & $\mathrm{~T}$ & $\mathrm{~S}$ & $\mathrm{~S}$ & $\mathrm{~S}$ & $\mathrm{~S}$ & $\mathrm{~S}$ & $\mathrm{~S}$ & $\mathrm{~S}$ & $\mathrm{~S}$ & $\mathrm{~S}$ & $\mathrm{~S}$ & $\mathrm{~S}$ & $\mathrm{~S}$ \\
\hline 289 & $\mathrm{C}$ & $\mathrm{F}$ & $\mathrm{F}$ & $\mathrm{F}$ & $\mathrm{F}$ & $\mathrm{F}$ & $\mathrm{F}$ & $\mathrm{F}$ & $\mathrm{F}$ & $\mathrm{F}$ & $\mathrm{F}$ & $\mathrm{F}$ & $\mathrm{F}$ & $\mathrm{F}$ \\
\hline 307 & G & A & G & G & G & G & G & G & G & G & G & G & G & G \\
\hline 309 & Q & $\mathrm{R}$ & $\mathrm{R}$ & $\mathrm{R}$ & $\mathrm{R}$ & $\mathrm{R}$ & $\mathrm{R}$ & $\mathrm{K}$ & $\mathrm{R}$ & $\mathrm{R}$ & $\mathrm{R}$ & $\mathrm{R}$ & $\mathrm{R}$ & $\mathrm{R}$ \\
\hline 324 & $\mathrm{C}$ & A & A & A & A & A & A & A & A & A & A & A & A & A \\
\hline 338 & A & A & A & A & $\mathrm{T}$ & A & A & A & A & A & A & A & A & A \\
\hline 380 & $\mathrm{R}$ & $\mathrm{K}$ & $\mathrm{K}$ & $\mathrm{K}$ & $\mathrm{K}$ & $\mathrm{K}$ & $\mathrm{K}$ & $\mathrm{K}$ & $\mathrm{K}$ & $\mathrm{K}$ & $\mathrm{K}$ & $\mathrm{K}$ & $\mathrm{K}$ & $\mathrm{K}$ \\
\hline 396 & A & V & A & A & A & A & A & A & A & A & A & A & A & A \\
\hline 400 & A & V & V & V & V & V & A & $\mathrm{V}$ & $\mathrm{V}$ & $\mathrm{T}$ & V & $\mathrm{V}$ & V & $\mathrm{V}$ \\
\hline 414 & M & V & V & V & $\mathrm{V}$ & I & V & V & V & $\mathrm{C}$ & V & V & V & V \\
\hline 419 & $\mathrm{~L}$ & I & I & I & I & I & I & I & I & I & I & I & I & I \\
\hline 421 & A & V & V & V & V & V & V & V & V & V & V & V & V & V \\
\hline 424 & I & I & V & I & I & V & V & V & I & V & V & I & V & V \\
\hline 432 & V & I & I & I & I & I & I & I & I & I & I & I & I & I \\
\hline 434 & I & Q & Q & $\mathrm{Q}$ & Q & Q & Q & Q & $\mathrm{Q}$ & Q & $\mathrm{Q}$ & Q & $\mathrm{Q}$ & Q \\
\hline 444 & $\mathrm{~N}$ & $\mathrm{D}$ & $\mathrm{D}$ & $\mathrm{D}$ & $\mathrm{D}$ & $\mathrm{D}$ & $\mathrm{D}$ & $\mathrm{D}$ & $\mathrm{D}$ & $\mathrm{D}$ & $\mathrm{D}$ & $\mathrm{D}$ & $\mathrm{D}$ & $\mathrm{D}$ \\
\hline 445 & $\mathrm{C}$ & $\mathrm{F}$ & $\mathrm{F}$ & $\mathrm{F}$ & F & $\mathrm{F}$ & $\mathrm{F}$ & $\mathrm{F}$ & $\mathrm{F}$ & $\mathrm{F}$ & $\mathrm{F}$ & $\mathrm{F}$ & $\mathrm{F}$ & $\mathrm{F}$ \\
\hline 446 & $\mathrm{E}$ & $\mathrm{D}$ & $\mathrm{D}$ & $\mathrm{D}$ & $\mathrm{D}$ & $\mathrm{D}$ & D & $\mathrm{D}$ & $\mathrm{D}$ & $\mathrm{D}$ & $\mathrm{D}$ & $\mathrm{D}$ & $\mathrm{D}$ & $\mathrm{D}$ \\
\hline 447 & I & $\mathrm{M}$ & $\mathrm{M}$ & I & $\mathrm{M}$ & $\mathrm{M}$ & $\mathrm{M}$ & $\mathrm{M}$ & M & $\mathrm{M}$ & M & $\mathrm{M}$ & $\mathrm{M}$ & M \\
\hline 450 & A & V & V & $\mathrm{V}$ & $\mathrm{V}$ & V & V & V & $\mathrm{V}$ & $\mathrm{V}$ & V & $\mathrm{V}$ & V & $\mathrm{V}$ \\
\hline 451 & $\mathrm{C}$ & $\mathrm{T}$ & $\mathrm{T}$ & $\mathrm{T}$ & $\mathrm{T}$ & $\mathrm{T}$ & $\mathrm{T}$ & $\mathrm{T}$ & $\mathrm{T}$ & $\mathrm{T}$ & $\mathrm{T}$ & $\mathrm{T}$ & $\mathrm{T}$ & $\mathrm{T}$ \\
\hline 455 & $\mathrm{E}$ & $\mathrm{T}$ & $\mathrm{T}$ & $\mathrm{T}$ & $\mathrm{T}$ & $\mathrm{T}$ & $\mathrm{T}$ & $\mathrm{T}$ & $\mathrm{T}$ & $\mathrm{T}$ & $\mathrm{T}$ & $\mathrm{T}$ & $\mathrm{T}$ & $\mathrm{T}$ \\
\hline 480 & G & $\mathrm{H}$ & $\mathrm{H}$ & $\mathrm{H}$ & $\mathrm{H}$ & $\mathrm{H}$ & $\mathrm{H}$ & $\mathrm{H}$ & $\mathrm{H}$ & $\mathrm{H}$ & $\mathrm{H}$ & $\mathrm{H}$ & $\mathrm{H}$ & $\mathrm{H}$ \\
\hline 482 & I & $\mathrm{L}$ & $\mathrm{L}$ & $\mathrm{L}$ & $\mathrm{L}$ & $\mathrm{L}$ & $\mathrm{L}$ & $\mathrm{L}$ & $\mathrm{L}$ & $\mathrm{L}$ & $\mathrm{L}$ & $\mathrm{L}$ & $\mathrm{L}$ & $\mathrm{L}$ \\
\hline 487 & A & $\mathrm{G}$ & $\mathrm{G}$ & $\mathrm{G}$ & $\mathrm{G}$ & $\mathrm{G}$ & $\mathrm{G}$ & $\mathrm{G}$ & $\mathrm{G}$ & $\mathrm{G}$ & $\mathrm{G}$ & $\mathrm{G}$ & $\mathrm{G}$ & G \\
\hline 488 & $\mathrm{C}$ & A & $\mathrm{S}$ & $\mathrm{C}$ & $\mathrm{S}$ & $\mathrm{S}$ & $\mathrm{S}$ & $\mathrm{S}$ & $S$ & A & $\mathrm{S}$ & $\mathrm{S}$ & $\mathrm{S}$ & $S$ \\
\hline 534 & $\mathrm{~L}$ & $\mathrm{~L}$ & $\mathrm{~L}$ & $\mathrm{~L}$ & $\mathrm{~L}$ & $\mathrm{~L}$ & $\mathrm{P}$ & $\mathrm{L}$ & $\mathrm{L}$ & $\mathrm{L}$ & $\mathrm{L}$ & $\mathrm{L}$ & $\mathrm{L}$ & $\mathrm{L}$ \\
\hline 539 & I & $\mathrm{L}$ & $\mathrm{L}$ & $\mathrm{L}$ & $\mathrm{L}$ & $\mathrm{L}$ & $\mathrm{L}$ & $\mathrm{L}$ & $\mathrm{L}$ & $\mathrm{L}$ & $\mathrm{L}$ & $\mathrm{L}$ & $\mathrm{L}$ & $\mathrm{L}$ \\
\hline 540 & A & $\mathrm{P}$ & $\mathrm{P}$ & $\mathrm{P}$ & $\mathrm{P}$ & $\mathrm{P}$ & $\mathrm{P}$ & $\mathrm{P}$ & $\mathrm{P}$ & $\mathrm{P}$ & $\mathrm{P}$ & $\mathrm{P}$ & $\mathrm{P}$ & $\mathrm{P}$ \\
\hline 543 & $\mathrm{G}$ & A & A & A & A & A & A & A & A & A & A & A & A & A \\
\hline 544 & $\mathrm{R}$ & $\mathrm{K}$ & $\mathrm{K}$ & $\mathrm{K}$ & $\mathrm{K}$ & $\mathrm{K}$ & $\mathrm{K}$ & $\mathrm{K}$ & $\mathrm{K}$ & $\mathrm{K}$ & $\mathrm{K}$ & $\mathrm{K}$ & $\mathrm{K}$ & $\mathrm{K}$ \\
\hline 549 & $\mathrm{G}$ & $\mathrm{G}$ & $\mathrm{G}$ & $\mathrm{G}$ & $\mathrm{G}$ & $\mathrm{S}$ & $\mathrm{S}$ & $\mathrm{G}$ & $\mathrm{G}$ & $\mathrm{G}$ & $\mathrm{G}$ & $\mathrm{G}$ & $\mathrm{G}$ & $\mathrm{G}$ \\
\hline 552 & $\mathrm{~T}$ & $\mathrm{~T}$ & $\mathrm{~K}$ & $\mathrm{~T}$ & $\mathrm{~T}$ & $\mathrm{~T}$ & $\mathrm{~T}$ & $\mathrm{~T}$ & $\mathrm{~T}$ & $\mathrm{~K}$ & $\mathrm{~K}$ & $\mathrm{~T}$ & $\mathrm{~T}$ & $\mathrm{~K}$ \\
\hline 553 & A & V & V & V & V & V & V & V & V & V & V & V & V & V \\
\hline 555 & $\mathrm{Y}$ & A & A & A & A & A & A & A & A & A & A & A & A & A \\
\hline 556 & $\mathrm{~S}$ & $\mathrm{G}$ & $\mathrm{G}$ & $\mathrm{G}$ & $\mathrm{G}$ & $\mathrm{G}$ & $\mathrm{G}$ & $\mathrm{G}$ & $\mathrm{G}$ & $\mathrm{G}$ & $\mathrm{G}$ & $\mathrm{G}$ & $\mathrm{G}$ & $\mathrm{G}$ \\
\hline 557 & $\mathrm{G}$ & $\mathrm{G}$ & $\mathrm{G}$ & $\mathrm{G}$ & $\mathrm{G}$ & G & $\mathrm{G}$ & $\mathrm{G}$ & $\mathrm{G}$ & $\mathrm{G}$ & $\mathrm{G}$ & $\mathrm{G}$ & $\mathrm{E}$ & $\mathrm{G}$ \\
\hline 564 & V & $\mathrm{M}$ & V & M & M & M & $\mathrm{M}$ & M & M & M & M & $\mathrm{M}$ & M & V \\
\hline 566 & $\mathrm{H}$ & $\mathrm{H}$ & $\mathrm{H}$ & $\mathrm{H}$ & $\mathrm{H}$ & $\mathrm{H}$ & $\mathrm{H}$ & $\mathrm{H}$ & $\mathrm{H}$ & $\mathrm{H}$ & $\mathrm{H}$ & $\mathrm{R}$ & $\mathrm{R}$ & $\mathrm{R}$ \\
\hline 571 & W & $\mathrm{Y}$ & $\mathrm{Y}$ & $\mathrm{Y}$ & $\mathrm{Y}$ & $\mathrm{Y}$ & $\mathrm{Y}$ & $\mathrm{Y}$ & $\mathrm{Y}$ & $\mathrm{Y}$ & $\mathrm{Y}$ & $\mathrm{Y}$ & $\mathrm{Y}$ & $\mathrm{Y}$ \\
\hline 572 & $\mathrm{~F}$ & $\mathrm{~L}$ & $\mathrm{~L}$ & $\mathrm{~L}$ & $\mathrm{~L}$ & $\mathrm{~L}$ & $\mathrm{~L}$ & $\mathrm{~L}$ & $\mathrm{~L}$ & $\mathrm{~L}$ & $\mathrm{~L}$ & $\mathrm{~F}$ & $\mathrm{~L}$ & $\mathrm{~L}$ \\
\hline 573 & W & $\mathrm{L}$ & $\mathrm{L}$ & $\mathrm{L}$ & $\mathrm{L}$ & $\mathrm{L}$ & $\mathrm{L}$ & $\mathrm{L}$ & $\mathrm{L}$ & $\mathrm{L}$ & $\mathrm{L}$ & $\mathrm{L}$ & $\mathrm{L}$ & $\mathrm{L}$ \\
\hline
\end{tabular}


Table 2 (continued)

\begin{tabular}{|c|c|c|c|c|c|c|c|c|c|c|c|c|c|c|}
\hline \multicolumn{2}{|c|}{$\begin{array}{l}\text { 1a. H77 } \\
\text { NC_004102 }\end{array}$} & $\begin{array}{l}4 a \\
\text { ED43 } \\
\text { Y11604 }\end{array}$ & $\begin{array}{l}\text { EG } \\
\text { DAM } \\
083\end{array}$ & $\begin{array}{l}\text { EG } \\
\text { DAM } \\
087\end{array}$ & $\begin{array}{l}\text { EG } \\
\text { DAM } \\
093\end{array}$ & $\begin{array}{l}\text { EG } \\
\text { DAM } \\
094\end{array}$ & $\begin{array}{l}\text { EG } \\
\text { DAM } \\
095\end{array}$ & $\begin{array}{l}\text { EG } \\
\text { DAM } \\
103\end{array}$ & $\begin{array}{l}\text { EG } \\
\text { DAM } \\
105\end{array}$ & $\begin{array}{l}\text { EG } \\
\text { DAM } \\
108\end{array}$ & $\begin{array}{l}\text { EG } \\
\text { DAM } \\
113\end{array}$ & $\begin{array}{l}\text { EG } \\
\text { DAM } \\
115\end{array}$ & $\begin{array}{l}\text { EG } \\
\text { DAM } \\
122\end{array}$ & $\begin{array}{l}\text { EG } \\
\text { DAM } \\
126\end{array}$ \\
\hline 574 & $\mathrm{~F}$ & $\mathrm{~L}$ & $\mathrm{~L}$ & $\mathrm{~L}$ & $\mathrm{~L}$ & $\mathrm{~L}$ & $\mathrm{~L}$ & $\mathrm{~L}$ & $\mathrm{~L}$ & $\mathrm{~L}$ & $\mathrm{~L}$ & $\mathrm{~L}$ & $\mathrm{~L}$ & $\mathrm{~L}$ \\
\hline 578 & $\mathrm{~L}$ & I & $\mathrm{L}$ & $\mathrm{L}$ & $\mathrm{L}$ & $\mathrm{L}$ & $\mathrm{L}$ & $\mathrm{L}$ & $\mathrm{L}$ & $\mathrm{L}$ & $\mathrm{L}$ & $\mathrm{L}$ & $\mathrm{L}$ & $\mathrm{L}$ \\
\hline 580 & A & $\mathrm{T}$ & $\mathrm{S}$ & S & $\mathrm{S}$ & S & $\mathrm{S}$ & $\mathrm{S}$ & $S$ & $S$ & $S$ & $S$ & $\mathrm{~S}$ & S \\
\hline 581 & A & V & V & V & V & V & $\mathrm{V}$ & V & V & $\mathrm{V}$ & V & V & $\mathrm{V}$ & V \\
\hline 586 & $\mathrm{Y}$ & $\mathrm{F}$ & $\mathrm{F}$ & $\mathrm{F}$ & $\mathrm{F}$ & $\mathrm{F}$ & $\mathrm{F}$ & $\mathrm{F}$ & $\mathrm{F}$ & $\mathrm{F}$ & $\mathrm{F}$ & $\mathrm{F}$ & $\mathrm{F}$ & $\mathrm{F}$ \\
\hline 590 & $\mathrm{~N}$ & A & A & A & A & A & A & A & A & A & A & A & A & A \\
\hline
\end{tabular}

Only the important amino acids are shown compared to the amino acids of the reference prototype 1a (NC_004102) and the Egyptian reference subtype 4a (Y11604)

acid (aa) variance in the NS5B region of the twelve isolates under study revealed the presence of several substitutions (Table 2 and supplementary Fig. S1). Several substitutions in the Egyptian reference Y11604 were not recorded in the twelve studied isolates, e.g. H63T, A80P, A105K, N120S, E128D, V167S, L182T, P183A, E258D, S282T, G307A, A396V, and L578I. In addition, some substitutions dominated in the isolates of this study but did not exist in the reference Y11604; S62N, K124E, K181Q and L184E.

\section{Variability of the full length of NS3-4A region}

Compared with the Egyptian reference subtype 4a (Y11604) and the reference prototype 1a (NC_004102), aa variance analysis in the NS3-4A region of the studied isolates revealed the presence of several substitutions (Table 3 and supplementary Fig. S2). Several substitutions in the Egyptian reference Y11604 were not recorded in the 12 studied isolates, e.g. S102A, Y218H, H293Y, V318T, T448I, S489A, V490E, N518D, C568S, A573L, P574A, and $\mathrm{T} 610 \mathrm{~V}$. In addition, some substitutions dominated in the isolates under study but did not exist in the reference Y11604; S101A, I114V, M485I/L and H541Q.

\section{Discussion}

In the current study, RFLP technique was used for genotyping $80 \mathrm{HCV}$ samples from Egyptian patients by double restriction digestion using MvaI/HinfI and RsaI/HaeIII enzymatic combinations. The typical cleavage patterns of MvaI/Hinfl and RsaI/HaeIII for HCV type 4 and type 1 were reported in a previous study [4]. Irregular cleavage patterns were observed with some samples in the current study because of the absence of some restriction enzyme sites or creation of extra sites. An irregular type $1 \mathrm{Mval} /$ Hinfl cleavage pattern; 69, 56, 129 and $177 \mathrm{bp}$; was seen with sample 090 because of the failure to cut some PCR products in one $M v a \mathrm{I}$ site. Out of the $80 \mathrm{HCV}$ samples, five samples (6\%) were determined to be HCV type 1, while the other 75 samples were determined to be HCV type 4 (94\%). Based on RFLP results, HCV genotype 4 is the predominant genotype in these samples followed by genotype 1 , a result that is consistent with other studies $[4,6,17]$.

Because of misidentification of HCV subtypes by $5^{\prime}$ UTR-based RFLP [18], the use of one of the most divergent $\mathrm{HCV}$ regions; core, E1 and NS5B, became a useful subtyping tool [19]. Sequencing a portion of NS5B region followed by phylogenetic analysis became the common and standard method for identifying HCV genotypes and subtypes [20]. This technique, however, cannot clearly distinguish between some HCV genotypes, so HCV subtyping based on sequencing the full-length of NS5B is considered more accurate than partial NS5B sequencing [21]. In the current study, twelve HCV samples were sequenced for the full length of their NS5B and were analyzed using phylogenetic analysis. Neighbor joining phylogenetic tree revealed that these twelve sequenced samples fall in subtype $4 \mathrm{a}$ clade, and cluster with each other and with HCV subtype 4a from Egypt and USA. Overall, the results of the current study revealed that $\mathrm{HCV}$ subtype $4 \mathrm{a}$ is the predominant subtype, which is consistent with other studies in different governorates in Egypt [4, 5, 22, 23].

HCV NS5B is the RNA-dependent RNA polymerase (RdRp) [24] responsible for viral genome replication. HCV polymerase replication activity [25] and treatment outcomes [26] are affected by aa substitutions in the NS5B polymerase region. Based on NS5B polymerase 3D structure, this enzyme consists of three domains: Palm, Fingers, and Thumb [27]. The residues 188-227 and 287-370 form the palm domain. The thumb domain extends from aa 371 to aa 563 [28]. In the twelve isolates of the current study and in the Egyptian reference genotype $4 \mathrm{a}$ (Y11604), the active site residues D220, D225, G317, D318, and D319 are conserved. Residues G283, T286, T287, and N291 responsible for sugar selection by NS5B [29] are also conserved. The residues R158, S367, R386, and R394 which are important for NTPs interaction are also conserved. The residues $360-370$ that 
Table 3 Amino acid variance in NS3-4A region of $12 \mathrm{HCV}$ isolates

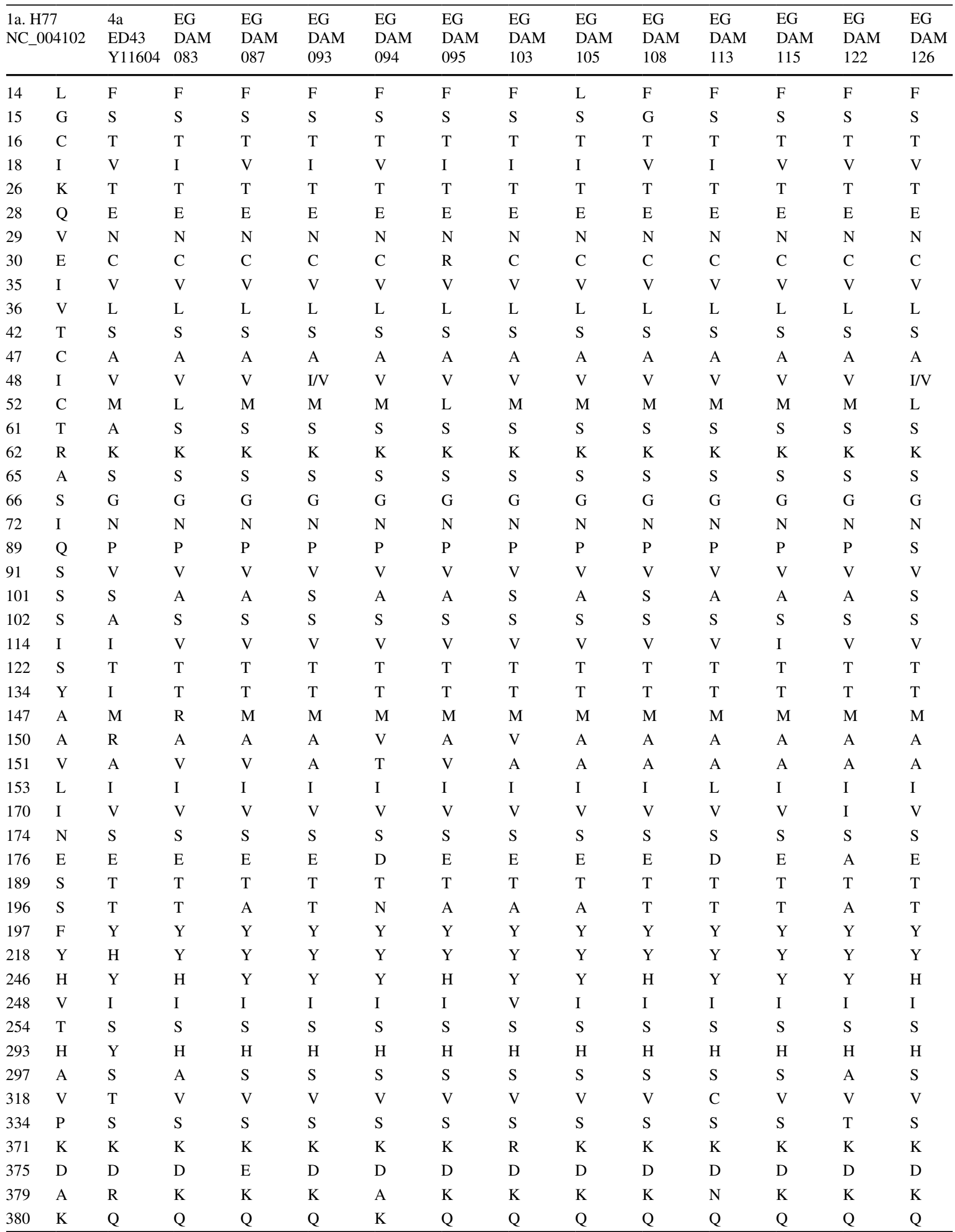


Table 3 (continued)

\begin{tabular}{|c|c|c|c|c|c|c|c|c|c|c|c|c|c|c|}
\hline \multicolumn{2}{|c|}{$\begin{array}{l}\text { 1a. H77 } \\
\text { NC_004102 }\end{array}$} & $\begin{array}{l}4 a \\
\text { ED43 } \\
\text { Y11604 }\end{array}$ & $\begin{array}{l}\text { EG } \\
\text { DAM } \\
083\end{array}$ & $\begin{array}{l}\text { EG } \\
\text { DAM } \\
087\end{array}$ & $\begin{array}{l}\text { EG } \\
\text { DAM } \\
093\end{array}$ & $\begin{array}{l}\text { EG } \\
\text { DAM } \\
094\end{array}$ & $\begin{array}{l}\text { EG } \\
\text { DAM } \\
095\end{array}$ & $\begin{array}{l}\text { EG } \\
\text { DAM } \\
103\end{array}$ & $\begin{array}{l}\text { EG } \\
\text { DAM } \\
105\end{array}$ & $\begin{array}{l}\text { EG } \\
\text { DAM } \\
108\end{array}$ & $\begin{array}{l}\text { EG } \\
\text { DAM } \\
113\end{array}$ & $\begin{array}{l}\text { EG } \\
\text { DAM } \\
115\end{array}$ & $\begin{array}{l}\text { EG } \\
\text { DAM } \\
122\end{array}$ & $\begin{array}{l}\text { EG } \\
\text { DAM } \\
126\end{array}$ \\
\hline 382 & $\mathrm{~V}$ & $\mathrm{~T}$ & $\mathrm{~T}$ & $\mathrm{~T}$ & $\mathrm{~T}$ & $\mathrm{~T}$ & $\mathrm{~T}$ & $\mathrm{~T}$ & $\mathrm{~T}$ & $\mathrm{~T}$ & $\mathrm{R}$ & $\mathrm{T}$ & $\mathrm{T}$ & $\mathrm{T}$ \\
\hline 386 & I & $\mathrm{L}$ & $\mathrm{L}$ & $\mathrm{L}$ & $\mathrm{L}$ & $\mathrm{L}$ & $\mathrm{L}$ & $\mathrm{L}$ & $\mathrm{L}$ & $\mathrm{L}$ & $\mathrm{L}$ & $\mathrm{L}$ & $\mathrm{L}$ & $\mathrm{L}$ \\
\hline 410 & $\mathrm{~S}$ & A & A & A & A & A & A & A & A & A & A & A & A & A \\
\hline 445 & $\mathrm{~T}$ & S & $S$ & $S$ & S & $\mathrm{S}$ & S & $S$ & S & $\mathrm{S}$ & S & $S$ & S & $S$ \\
\hline 448 & $\mathrm{~T}$ & I & $\mathrm{T}$ & $\mathrm{T}$ & $\mathrm{T}$ & $\mathrm{T}$ & $\mathrm{T}$ & $\mathrm{T}$ & $\mathrm{T}$ & $\mathrm{T}$ & $\mathrm{T}$ & $\mathrm{T}$ & $\mathrm{T}$ & $\mathrm{T}$ \\
\hline 459 & $\mathrm{~T}$ & S & S & S & S & S & S & S & S & S & S & S & S & $\mathrm{S}$ \\
\hline 470 & $\mathrm{P}$ & $\mathrm{L}$ & $\mathrm{L}$ & $\mathrm{L}$ & $\mathrm{L}$ & $\mathrm{L}$ & $\mathrm{L}$ & $\mathrm{L}$ & $\mathrm{L}$ & $\mathrm{L}$ & $\mathrm{L}$ & $\mathrm{L}$ & $\mathrm{L}$ & $\mathrm{L}$ \\
\hline 485 & M & M & I & $\mathrm{M}$ & $\mathrm{I} / \mathrm{M}$ & I & I & I & I & I & I & I & $\mathrm{L}$ & I \\
\hline 489 & $\mathrm{~S}$ & A & S & S & S & $\mathrm{S}$ & $\mathrm{S}$ & S & S & S & S & S & $\mathrm{S}$ & S \\
\hline 490 & $\mathrm{~V}$ & E & V & V & V & $\mathrm{V}$ & V & V & V & V & V & V & V & V \\
\hline 511 & V & $\mathrm{T}$ & $\mathrm{T}$ & $\mathrm{T}$ & $\mathrm{T}$ & $\mathrm{T}$ & I & V & $\mathrm{T}$ & $\mathrm{T}$ & $\mathrm{T}$ & $\mathrm{T}$ & $\mathrm{T}$ & $\mathrm{T}$ \\
\hline 514 & $\mathrm{R}$ & $\mathrm{K}$ & $\mathrm{R}$ & $\mathrm{R}$ & $\mathrm{R}$ & $\mathrm{R}$ & $\mathrm{R}$ & $\mathrm{R}$ & $\mathrm{R}$ & $\mathrm{R}$ & $\mathrm{K}$ & $\mathrm{R}$ & $\mathrm{R}$ & $\mathrm{R}$ \\
\hline 518 & $\mathrm{~N}$ & $\mathrm{D}$ & $\mathrm{N}$ & $\mathrm{N}$ & $\mathrm{N}$ & $\mathrm{N}$ & $\mathrm{N}$ & $\mathrm{N}$ & $\mathrm{N}$ & $\mathrm{N}$ & $\mathrm{N}$ & $\mathrm{N}$ & $\mathrm{N}$ & $\mathrm{N}$ \\
\hline 541 & $\mathrm{H}$ & $\mathrm{H}$ & Q & Q & $\mathrm{Q}$ & $\mathrm{Q}$ & $\mathrm{H}$ & Q & Q & $\mathrm{Q}$ & $\mathrm{Q}$ & Q & $\mathrm{Q}$ & $\mathrm{H}$ \\
\hline 568 & $\mathrm{C}$ & $S$ & $\mathrm{C}$ & $\mathrm{C}$ & $\mathrm{C}$ & $\mathrm{C}$ & $\mathrm{C}$ & $\mathrm{C}$ & $\mathrm{C}$ & $\mathrm{C}$ & $\mathrm{C}$ & $\mathrm{C}$ & $\mathrm{C}$ & $\mathrm{C}$ \\
\hline 573 & A & L & A & A & A & A & A & A & A & $\mathrm{H}$ & A & A & A & A \\
\hline 574 & $\mathrm{P}$ & A & $\mathrm{P}$ & $\mathrm{P}$ & $P$ & $\mathrm{P}$ & $\mathrm{P}$ & $\mathrm{P}$ & $\mathrm{P}$ & $\mathrm{P}$ & $\mathrm{P}$ & $\mathrm{P}$ & $\mathrm{P}$ & $\mathrm{P}$ \\
\hline 586 & I & I & I & $\mathrm{N}$ & I & I & I & I & I & I & $\mathrm{T}$ & I & I & I \\
\hline 610 & $\mathrm{~T}$ & V & $\mathrm{T}$ & $\mathrm{T}$ & $\mathrm{T}$ & $\mathrm{T}$ & $\mathrm{T}$ & $\mathrm{T}$ & $\mathrm{T}$ & $\mathrm{T}$ & $\mathrm{T}$ & $\mathrm{T}$ & $\mathrm{T}$ & $\mathrm{E}$ \\
\hline 678 & $\mathrm{E}$ & Q & Q & Q & Q & $\mathrm{Q}$ & $Q$ & Q & Q & $\mathrm{H}$ & Q & Q & $Q$ & Q \\
\hline
\end{tabular}

Only the important amino acids are shown compared to the amino acids of the reference prototype 1a (NC_004102) and the Egyptian reference subtype 4a (Y11604)

form motif $\mathrm{E}$ of the palm domain [28] and are important for maintaining proper secondary structure for the right palm and thumb domains positioning [30] are also conserved. The residues E18, Y191, C274, Y276, and H502 that take part in primer-template interaction [31] are conserved too except for E18 which was substituted into $\mathrm{G}$ in four isolates (EGDAM087, EGDAM093, EGDAM103 and EGDAM115). Absence of aa substitutions in these residues confirms the conservation of $\mathrm{HCV}$ palm domain and suggests normal polymerase activity in HCV Egyptian isolates.

Fingers domain contains two motifs and two functional regions [27]. Motif F (aa 132-162) is located near the template channel and involved in NTPs and RNA binding [32]. Motif G (aa 95-99) is important for primer-dependent RNA synthesis but not important for enzyme-template binding [33]. In the current study, six aa substitutions at positions $135,146,148,149,150,156$, and 162 were detected in motif F. In motif G, only one aa substitution was detected at position 98 and detected in nine isolates. Some substitutions in motif $\mathrm{F}$ were solo detected and some of the amino acids in motif $\mathrm{F}$ and $\mathrm{G}$ were substituted into other amino acids of the same or different type, therefore further investigation is mandatory to determine whether these mutations affect NTPs and RNA binding or not. Both functional regions I (aa 91-94) and II (aa 168-183) play a role in template binding [30]. In the twelve isolates of the current study, the functional region I was conserved while the functional region II was mutated. Six substitutions at positions $173,176,178,179,180$, and 181 were detected in the functional region II. Two aa substitutions at positions 182 and 183 appeared in the Egyptian reference genotype $4 \mathrm{a}$ (Y11604). Some of these aa substitutions are a change into aa of the same type, while others are a change into non-polar aa, a change from non-polar into a positively charged aa, and a change from a hydrophobic into hydrophilic aa. The presence of these substitutions could affect template-binding affinity in these isolates.

A unique feature of HCV NS5B is the $\beta$ hairpin loop (aa 443-455) that presents within the thumb domain. It acts as a gate for the template and primer [34] and it is responsible for correct initiation by right $3^{\prime}$ terminus recognition [35]. In this loop seven aa substitutions at positions 444,445 , $446,447,450,451$, and 455 were detected in the currently studied isolates and in the Egyptian reference genotype 4a (Y11604). This is in agreement with Di Maio et al. [28] study where $\beta$ loop of the thumb domain was highly variable in all genotypes including genotype 4 . The current aa substitutions are from polar into non-polar aa, hydrophilic into hydrophobic and vice versa. Thus, the presence of 
these mutations could affect template and primer orientation toward the active site in these isolates.

Palm and fingers domains are connected through 34 residues (aa 230-263) forming two amphipathic $\alpha$-helices that are important for maintaining the helical structure [27]. These two helices are connected through bidentate salt bridge link, respectively R234 and Q241 on one helix with E258 and R250 on the other helix [27]. Seventeen aa substitutions were detected in the two helices among the twelve isolates of the current study. In the four amino acids forming the bidentate salt bridge, R234 and Q241 were detected in one isolate only. E258 was substituted into D only in the reference genotype 4a (Y11604) but not in the isolates of the current study. Changes in these residues included changes in nature (polarity, hydrophobicity, or negative charging), which may affect the conformation of the $\alpha$-helix in these isolates.

Fingers and thumb domains are connected through two loops. The longer loop (aa 11-45), located near the NS5B $\mathrm{N}$-terminus, and the shorter loop (aa 139-160). These two loops are essential for obtaining the unique HCV NS5B shape [27]. In these two loops, twelve aa substitutions (E18G, Q19S, N24S, A25P, L36M, S39A, S42T, C146A/V, Q148N, P149Q, E150A/T, and P156A) were observed in some isolates in the current study. The substitution of hydrophobic to a hydrophilic aa or vice versa in these isolates is likely to affect HCV NS5B unique shape in these isolates.

NS5B C-terminus residues T532-R570 are responsible for regulating polymerase activity [34]. In the isolates of the current study, the thirteen substitutions L534P, I539L, A540P, G543A, R544K, G549S, T552K, A553V, Y555A, S556G, G557E, V564M, and H566R were recorded. Comparison of different $\mathrm{HCV}$ genotypes with $\mathrm{HCV}$ subtype $1 \mathrm{~b}$ in [28] proved the presence of 22 aa substitutions in the region spanning the residues T532-R570, indicating a high variability in this region. Amino acids 571-591 in the NS5B C-terminal end form the membrane anchor [34]. In the isolates of the current study, eight substitutions (W571Y, F572L, W573L, F574L, A580S, A581 V, Y586F, and N590A) were detected in the membrane-anchor region. In these substitutions, amino acids changed from aromatic into aliphatic ones, from hydrophobic into hydrophilic ones or vice versa. Based on this, these aa substitutions are expected to affect the function of the 21 residues. In the isolates of the current study, no aa substitutions were recorded in the conserved LLLL residue (aa 576-579) within the membraneanchor residues. But in the Egyptian reference genotype 4a (Y11604) L578 was substituted into I.

Substitutions in the NS5B region could be natural polymorphisms that cause resistance against antiviral drugs or induced mutations by INF/RBV treatment [36]. In the NS5B region, some mutations were generated due to mutagenic effect of Ribavirin [37]. These mutations include
V85I, D244N, D310N, S326G, R345S, and K379N/R [37]. Although the studied isolates in the current study are from treatment-naïve patients, some of the INF/RBV-induced mutations were detected; D244N only was detected in four isolates of the current study. The other INF/RBV-induced mutations were not detected. In Venezuelan patients, D310N, D244N and T329I/V/A were detected among different genotypes [36]. Q309R is another NS5B aa mutation that is significantly frequent in patients with SVR or during end-of treatment response [38]. Eleven out of the twelve studied isolates had the Q309R substitution.

Mutations at the NS5B active site or at one single domain of the four allosteric domains may affect all drug interactions on the entire protein [39]. Mutations in the active site were reported to confer resistance to Sofosbuvir and Mericitabine. These mutations include S282T (common in all HCV genotypes) [40], L159F, E341D, V321A, I434M (in genotype 1a) and in genotype 2a T179A and M289L [41, 42]. In addition, Sofosbuvir failure in $\mathrm{HCV}$ genotype $1 \mathrm{~b}$-infected patients was associated with $\mathrm{C} 316 \mathrm{~N} / \mathrm{H} / \mathrm{F}$ [42]. The substitutions L159F, T179A, M289L, V321A, C316N/H/F, E341D and I434M associated with Sofosbuvir resistance were not detected in the current study. All the studied isolates in the current study were free of the S282T mutation that was claimed to be associated with Sofosbuvir resistance. Absence of in vivo Sofosbuvir resistance mutations may be a good indicator for better rates of response to Sofosbuvir among Egyptians. Recently, Sofosbuvir plus Ribavirin for twelve or 24 weeks was reported to be effective in treating both treatment-naïve and previously treated HCV genotype 4-infected patients [43].

Within HCV NS5B, the allosteric sites are the targets of non-nucleoside inhibitors (NNIs) (Thumb I, Thumb II, Palm I, Palm II, and Palm- $\beta$ hairpin inhibitors) [44]. Substitutions associated with the resistance to NNI-I (thumb-I inhibitors) antiviral drugs (Deleobuvir, Beclabuvir, BILB 1941, and TMC647055) include P495L/S/T/A, P496S/T/A, V499A, A338V, A442T, I424V, A421V, and L392I. Both of the substitutions $\mathrm{P} 495 \mathrm{~L}$ and $\mathrm{A} 421 \mathrm{~V}$ were detected in HCV genotype 1 in a clinical trial of Beclabuvir [45]. The aa mutations (A338V, I424V, L392I, A442T, and V499A) associated with the resistance to Beclabuvir were detected in HCV genotype 1b-chronically infected treatment-naïve Chinese patients [46]. In the current study, A338 was substituted into T in one isolate (EGDAM093) and the substitution I424V was detected in eight of the twelve studied isolates. Both the reference sequence (Y11604) and the current isolates have the substitutions A421V and V499A. It is not known whether these aa substitutions can confer these isolates resistance to NNI-I.

Resistance to NNI-II (thumb-II inhibitors) was reported to be associated with L419M/S, R422K, M423T/I/S, M426A/T, I482L/S, A486V and V499A aa substitutions 
[47]. Both the substitutions L419I and I482L were observed in both the reference sequence (Y11604) and in the isolates of the current study. The presence of these two mutations may be a risk for potential resistance of subtype 4a-infected Egyptian patients to some thumb II inhibitors.

Presence of the substitutions C316Y/N, S368T/A, M414T/L, Y448H/C, G554D, Y555H/G, S556G, and D559G was associated with resistance to different palm I inhibitors (NNI-III) [15, 48]. This class of inhibitors targets the palm domain at the junction between palm and thumb domains and close to the active site [49]. In the current study, some aa mutations were detected in both the reference Y11604 and in the isolates of the current study. The residue M414 was mutated into V in the reference Y11604 and in 10 isolates of the current study, mutated into I in the isolate EGDAM094, and into C in the isolate EGDAM108. M414 was substituted into other non-polar hydrophobic (V or I) aa or into other polar hydrophilic aa (C). Both the reference Y11604 and the isolates of the current study have the substitution Y555A. The substitution S556G existed in the reference Y11604 and all isolates of the current study. Mutations at these residues may be a risk for potential resistance of these isolates to palm I inhibitors.

The substitutions L314F, C316Y, I363V, S365L, and M414V were reported to confer resistance to Nesbuvir in vitro [50]. Nesbuvir binds to palm II site of HCV RdRp and shows an in vitro inhibiting activity for HCV RdRp [50]. The substitution M414V existed in the reference Y11604 and ten isolates of the current study. M414 was mutated into $\mathrm{I}$ and $\mathrm{C}$ in the isolates EGDAM094 and EGDAM108, respectively.

Currently, Sofosbuvir that targets HCV NS5B region is massively used to treat Egyptian HCV patients since a few years. Because of the short period of Sofosbuvir use, it cannot be confirmed that all the treated patients will not show disease relapse. In addition to this, some $\mathrm{HCV}$ patients cannot receive Sofosbuvir treatment because of their liver or kidney clinical profiles. For these reasons the current study refocuses on studying the important viral region NS3-4A.

As a result of the high-HCV replication rate, and the poor HCV polymerase's proofreading, highly variable viruses are generated with substitutions in their genome to reduce susceptibility to antiviral agents [51]. NS3-4A region is translated to produce two non-structural $\mathrm{HCV}$ proteins NS3 protein (631 aa) and NS4A protein (54 aa) [52]. The NS3 protein has a protease domain (180 aa) in its $\mathrm{N}$ terminus and a helicase domain (450 aa) at the C terminus [53]. NS3 serine protease is responsible for cleavage at four sites (NS3/ NS4A, NS4A/NS4B, NS4B/NS5A, and NS5A/NS5B) in HCV polyprotein [54]. This enzyme consists of two domains $(\mathrm{N}-$ and $\mathrm{C}$-terminal domains) incorporating a groove containing the catalytic active site (H57, D81, and S139) [55]. Any substitution in the catalytic triad residues abolishes
NS3 protease role in cleavage of $\mathrm{HCV}$ polyprotein [56]. If S139 is mutated into A, cleavage process at the NS3/NS4A, NS4A/NS4B, NS4B/NS5A, and NS5A/NS5B junctions will be abolished [57]. At the position 81, D (acidic charged aa) mutated into $\mathrm{G}$ (hydrophobic non-polar aa) caused chemical disturbance of the enzyme [56]. NS3 catalytic triad residues (H57, D81, and S139) are conserved among the isolates of the current study indicating typical NS3 protease structure and activity.

Unlike extracellular serine proteases, NS3 serine protease does not have disulfide bridges but it has a zinc ion located at one $\beta$ barrel of the $\mathrm{C}$-terminal domain [55]. The zinc ion is tetrahyderally coordinated with 4 conserved residues, C97, C99, C145, and H149, forming the NS3 binding site [58]. NS3 zinc binding site is located opposite to the NS3 protease catalytic triad [59]. In the isolates of the current study, the residues $\mathrm{C} 97, \mathrm{C} 99, \mathrm{C} 145$, and $\mathrm{H} 149$ were conserved confirming the stable NS3 protease structure which ensures normal viral activity.

Mutations in positions close to catalytic site residues or close to residues that bind with zinc ion may affect NS3 3D structure which, in turn, may affect protease activity.

In Vallet et al. [56] study on subtype 1a or 1b patients, the substitutions Q86H, C97R, S174D, and P131H in NS3 region were detected. Inconsistent with Vallet et al. [56], none of these substitutions were detected in our isolates supporting a stable $3 \mathrm{D}$ structure and potentially an effective protein.

HCV NS3 helicase consists of three structural domains separated by clefts and these domains have conserved motifs that play different roles in NTP-binding, in NTPhydrolysis, and in nucleic acid binding [53].

Motif II (Walker motif B) of NS3 helicase domain I contains a conserved sequence DECH [53]. In Heilek and Peterson [60] study, the mutation of H293 into A in DECH of motif II did not affect ATPase activity, but abolished helicase activity. Inconsistent to this result, Kim et al. [61] approved that mutation at $\mathrm{H} 293$ decreases ATPase activity and has a small effect on helicase activity. The Egyptian reference Y11604 has the substitution H293Y. The twelve isolates of this study have $\mathrm{H}$ at the position 293 indicating potential proper helicase activity.

Domain II contains the conserved sequence Q460RXGRXGR which is needed for ATP hydrolysis [62]. When Q460 mutated into H, R464 mutated into A and R467 mutated into $\mathrm{K}$, a significant decrease in ATPase activity was observed [62]. The mutations Q460H, R464A and $\mathrm{R} 467 \mathrm{~K}$ were not detected in both the reference Y11604 and our isolates.

Two conserved motifs are found only in HCV helicase; Arg-clamp and the Phe-loop [63]. The Arg-clamp spans aa 392-397. R393 clamps the nucleic acid to the protein both in the presence and absence of ATP. When R393 is mutated 
into A; the protein will be able to bind nucleic acid and stimulate ATP hydrolysis, but the enzyme will fall from the nucleic acid during translocation before unwinding [63]. The second conserved motif, Phe-loop, spans aa 437-444 [63]. Phe-loop plays a structural role as it acts as a contact to link domains 2 and 3 as the two conserved residues F438 and F444 in this loop (in domain 2) pack into a pocket containing F531, F536 and W532 (in domain 3) [64]. Both the isolates of the current study and the reference Y11604 have non mutated R393, F531, W532, F536, F438, and F444.

W501 in domain 3 acts as a bookend to define the border of the substrate-binding cavity as the $3^{\prime}$ terminal base stacked against it [65]. In HCV genotype 1, substitution of W501 with a non-aromatic aa has been shown before to cause poor NS3-catalyzed nucleic acid unwinding [62] and block genotype $1 \mathrm{~b}$ replication in cells [66]. The reference Y11604 and the isolates of the current study do not have W501R indicating normal helicase activity among Egyptians.

NS3 serine protease's optimal activity requires binding of another viral protein; NS4A [67]. NS4A central region (aa 21-31) is required for NS3 serine protease activation [68]. Conserved residues (V23, I25, I29, and L31), especially I25, I/V29 of NS4A central region, are critical for optimal binding of NS4A with NS3 and for protease activation [69]. In the isolates of the current study, no aa mutations were detected in the conserved central NS4A region.

Resistance to Boceprevir and Telaprevir, which bond with the catalytic site of NS3 protease inhibitors, was associated with the substitutions V36L, T54S, V55I/A, I170V, and M175L in different HCV genotypes [70]. HCV patients infected with subtype $1 \mathrm{~g}$ who were non-responders to Boceprevir-based therapy, were reported to have the resistance aa variants T54S, P131S, and L135F [71]. In the current study, both the reference Y11604 and the isolates of the current study have the substitution V36L. At position 170, the reference Y11604 and our isolates have V instead of I except for isolate EGDAM122 which has I at this position. Combination of resistance aa mutations T54S and Q80K reduced the resistance to Boceprevir and Telaprevir when compared with the presence of T54S only [72]. While combination of the aa mutations at position 54 and 55 were reported to reduce the response to triple therapy containing Boceprevir [70]. Absence of these substitutions may predict the responsiveness of genotype 4a-infected Egyptian HCV patients to Boceprevir and Telaprevir treatments.

Simeprevir in vitro resistance was associated with Q80K polymorphism in treatment-naïve HCV genotype 1a-infected patients [73] and, also, it was associated with aa mutations at the positions $80,122,155,156$, and/or 168 in HCV genotype 4-infected patients [74]. In Scottish treatment-naïve population, aa mutations at positions 155,156 , and 168 were rare while Q80K mutation was common [75]. The absence of such resistance mutations in the studied isolates may predict possible responsiveness of Egyptians to Simeprevir treatments.

Reduced antiviral activity of Grazoprevir was associated with the presence of single NS3 substitution including $\mathrm{Y} 56 \mathrm{H}, \mathrm{R} 155 \mathrm{~K}, \mathrm{~A} 156 \mathrm{G} / \mathrm{T} / \mathrm{V}$, and D168A/E/G/N/S/V/Y in genotype 1a replicons, with the presence of F43S, Y56F, V107I, A156S/T/V, and D168A/G/V in genotype 1b replicons, and with the presence of D168A/V in genotype 4 replicons [76]. D168V mutation conferred 313-fold resistance to Paritaprevir. The resistance degree associated with this mutation in subtype $4 \mathrm{a}$ and $4 \mathrm{~d}$ was the same [77]. In HCV genotype 1-infected patients, resistance to Paritaprevir was associated with the presence of aa mutations at positions 155,156 , and 168 in vitro or after monotherapy [78]. The absence of these aa mutations may predict a possible responsiveness of Egyptians to both Grazoprevir and Paritaprevir.

After implementing PEG-INF/RBV treatments in Egypt for a long time, new treatment protocols were applied because of drawbacks of the IFN/RBV treatment [79]. The current applied HCV treatment protocols for Egyptian $\mathrm{HCV}$-infected patients are Sofosbuvir and Ribavirin for twelve or 24 weeks [43], Zepatier (Combination product of fixed-dose of Elbasvir and Grazoprevir) [80], Sofosbuvir and Daclatasvir without Ribavirin [13], and combinations of Sofosbuvir/Velpatasvir, Glecaprevir/Pibrentasvir and Sofosbuvir/Ledipasvir according to the guidelines of European Association for The Study of The Liver [11]. Sofosbuvir/Daclatasvir combination was considered safe and effective treatment regimen with sustained virological response reaching $96 \%$ at week twelve of treatment on HCV genotype 4-infected Egyptian patients [12]. The use of the combination Glecaprevir/Pibrentasvir for 8 weeks for treating non-cirrhotic HCV genotype 4-infected patients showed about 93\% SVR rate [81]. According to Shiha et al. [82], 8 weeks Ledipasvir/Sofosbuvir without Ribavirin regimen was highly effective for treatment-naïve patients with SVR rate about $95 \%$. The study of NS5B and NS3-4A regions, which are vital for the virus life cycle and considered as the most important drug targets in Egyptian isolates, revealed the variation of genotype 4a isolates in Egyptian patients. Further investigations on treated Egyptian HCV patients are needed to clearly evaluate the effectiveness of the newly introduced drugs and whether drug resistance is encountered in view of the results of the current study.

The effectiveness and response rates achieved by DAAs in treating HCV-infected patients are high [83], however, because of the presence of many asymptomatic and still undiagnosed and untreated patients, the failure of DAAs to protect against $\mathrm{HCV}$ reinfection [84], and the DAAs high cost [85], there is still an urgent need for $\mathrm{HCV}$ vaccine. The strategies of vaccines development focus on the induction of strong neutralizing antibodies (Nabs) and long-lasting 
responses of $\mathrm{CD} 4+$ and $\mathrm{CD} 8+\mathrm{T}$ cells [86]. One vaccine approach involved the use of the non-structural (NS) proteins that are the dominant targets for CD8+ T cells [87]. These proteins are considered relatively conserved compared to the envelope glycoproteins [86]. A vaccine composed of adenovirus vectors serotype 6 and chimpanzee adenovirus 3 (ChAd3) carrying NS proteins was tested in phase 1 clinical trial on healthy individuals. It succeeded in inducing polyfunctional specific CD4+ and CD8+ T cells and memory $\mathrm{CD} 8+\mathrm{T}$ cells [88]. This vaccine was further improved through ChAd 3 priming then boosting with a modified vaccinia vector; both steps express NS3-5 proteins. When tested in healthy volunteers, this vaccine showed optimal induction of cell-mediated immunity and it has been moved to phase 2 clinical trial as a prophylactic vaccine in high-risk people who inject drugs $[86,89]$.

Naeem and Waheed [90] predicted the highly conserved T-cell binding epitopes in the HCV NS3-4A as ideal targets for vaccine development and are able to induce strong immunity. In HCV NS3-4A, the T cell MHC I-specific epitopes were 157AVCTRGVAK, 208SGKSTKVPA, 266TYSTYGKFL and 367FCHSKKKCD. The T cell MHC II-specific epitopes were 267YSTYGKFLA, 300ILGIGTVLD and 536FTGLTHIDA. In addition, Ikram et al. [91] identified conserved specific epitopes from NS3-4A and NS5B. The T cell MHC I specific epitopes in the NS3-4A were 207GSGKSTKVP, 228LNPSVAATL, and 368HSKKKCDEL. The T cell MHC II epitopes in the NS3-4A were 300LGIGTVLDQ, 365IFCHSKKKC and 366FCHSKKKCD. The T cell MHC I epitopes in the NS5B were 136TTIMAKNEV, 146DLGVRVCEK and 219YDTRCFDST. The T cell MHC II epitopes in the NS5B were 137TIMAKNEVF and 275GYRRCRASGV. Ikram et al. [91] found that multiepitope vaccine, developed from combinations of these epitopes, can elicit specific immune responses against HCV. In the 12 isolates of current study, the majority of these NS3-4A and NS5B epitopes are conserved. Since most of $\mathrm{HCV}$ vaccine studies are based on genotype $1 \mathrm{a}$, the detailed characterization of HCV genotype 4a NS3-4A regions in the current study can be important for vaccine developers as genotype $4 \mathrm{a}$ infections constitute $15 \%$ of all $\mathrm{HCV}$ infections worldwide and they are the most common in North Africa and the Middle East [92].

Despite the success of DAAs in treating HCV, drug resistance, and possibility of occurrence of HCC in effectively treated chronic $\mathrm{HCV}$ patients are still problems facing DAAs treatment [93]. After HCV entry into hepatocytes, pattern recognition receptors recognize viral RNA causing production of type I and III interferons (IFNs). After activation by the secreted INFs, NK cells kill HCV-infected cells. Once $\mathrm{HCV}$ antigens are transferred to and processed and presented by DCs as peptides bound to MHCI and II, specific HCV $\mathrm{CD} 8$ and $\mathrm{CD} 4$ cell responses are primed. HCV-infected cells are then eliminated by $\mathrm{HCV}$-specific $\mathrm{CD} 8 \mathrm{~T}$ cells through cytolytic or non-cytolytic mechanisms [94]. The CD8 T cells responses targeting the $\mathrm{HCV}$ non-structural proteins were found to be immunodominant and correlated with spontaneous clearance. Several HCV NS3, NS4 and NS5B epitopes were determined to elicit CD8 responses [95-98]. Mutations in these epitopes impair their binding with MHC I leading to disability of CD8 $\mathrm{T}$ cells recognition which prevent or delay CD8 T cells responses and cause viral persistence [99] and progression of infection toward chronicity. Here, the failure of the immune response is represented by the functional exhaustion of $\mathrm{T}$ cells, which ends with $\mathrm{T}$ cells apoptosis and a decline in the virus-specific $T$ cells [100].

Thus, advanced $\mathrm{T}$ cell-based strategies against $\mathrm{HCV}$ were developed. Transduction and engineering of CD4+ and $\mathrm{CD} 8+\mathrm{T}$ cells against different HCV epitopes (e.g. NS3 1073-1081, NS5A 1992-2000 and NS5B 2594-2602) via $\mathrm{T}$ cell receptor (TCR) gene transfer confer antiviral and cytotoxic activity and produce cytokines (IFN- $\gamma$, IL-2 and TNF- $\alpha$ ) [101, 102]. Zhang et al. [103] engineered high affinity TCRs targets (HCV NS3 1406-1415 epitope) and constructed a molecule called high-affinity T-cell activation core (HATac) through the fusion of high affinity TCRs with a T-cell activation molecule (anti-CD3 single-chain variable fragment) and this molecule succeeded to redirect functional non-specific CD8 T cells to recognize and kill cells presenting HCV NS3 antigens. In the currently sequenced isolates, some amino acid substitutions in HCV NS3-4A and NS5B epitopes were detected. However, no amino acid substitutions were detected in the HCV NS3 1031-1039 (AYAQQTRGL) epitope in the currently sequenced isolates. HCV NS3 1611-1618 (LIRLKPTL) was mutated into L(N/T)RLKPTL in two isolates only. HCV NS3 1636-1643 (TLTHPITK) was found to be mutated into VLTHPITK only in the reference Y11604 but not in any of the currently sequenced isolates. Some of these epitopes may be investigated for the use in transducing and engineering $\mathrm{T}$ cells.

\section{Conclusion}

This study genotyped HCV circulating in a population of Egyptian patients and indicated that genotype 4a is the predominant genotype. The study further dissected NS5B and NS3-4A regions of this genotype to reveal the amino acid variations in these regions. The relation between amino acid substitutions and resistance to different drugs was discussed. The results are preliminary and may be important for predicting responsiveness or resistance of $\mathrm{HCV}$ genotype 4 to the currently available HCV drugs that target NS5B and NS3$4 \mathrm{~A}$ regions. Further investigations might determine which combination therapy protocol might be applied for treating $\mathrm{HCV}$ type 4a-infected patients to avoid drug resistance and 
disease relapse. Also, our results showed that majority of the previously predicted T-cell binding epitopes in NS3-4A and NS5B regions are conserved in the currently studied isolates which might be important for $\mathrm{HCV}$ vaccine development. In addition, we revealed some NS3 epitopes for potential use in transducing and engineering $\mathrm{T}$ cells against $\mathrm{HCV}$ epitopes.

Acknowledgements Authors are thankful to the Faculty of Science, Damietta University, for supporting this study and accommodating the experiments in the laboratory of Biotechnology and Physiology.

Author contributions RT and AG performed experiments and wrote the manuscript; $\mathrm{AG}$ and $\mathrm{HZ}$ designed the experiments and analyzed sequence data; $\mathrm{HZ}$ collected blood samples and revised the manuscript.

\section{Compliance with ethical standards}

Conflict of interest The authors declare no conflict of interest.

Ethical approval All procedures followed were in accordance with the ethical standards of Helsinki Declaration of 1975 and our institute's board has approved the study. All patients have given informed consent before sample collection.

Informed consent All authors agreed with the content of this manuscript and they have given explicit consent to submit and publish it.

\section{References}

1. World Health Organization (2018) Hepatitis C. https://www.who. int/en/news-room/fact-sheets/detail/hepatitis-c

2. Hedskog C, Bhardwaj N, Chang S, Parhy B, Martin R, Zeuzem S, Moreno C, Shafran S, Borgia SM, Hyland RH (2017) Identification of novel $\mathrm{HCV}$ genotype and subtypes in patients treated with sofosbuvir based regimens. Hepatology 66:121A-121A

3. Simmonds P, Bukh J, Combet C, Deléage G, Enomoto N, Feinstone S, Halfon P, Inchauspé G, Kuiken C, Maertens G (2005) Consensus proposals for a unified system of nomenclature of hepatitis C virus genotypes. Hepatology 42(4):962-973

4. El-Tahan RR, Ghoneim AM, Zaghloul H (2018) 5' UTR and NS5B-based genotyping of hepatitis $\mathrm{C}$ virus in patients from Damietta governorate Egypt. J Adv Res 10:39-47

5. Fakhr AE, Pourkarim MR, Maes P, Atta AH, Marei A, Azab M, Van Ranst M (2013) Hepatitis C virus NS5B sequence-based genotyping analysis of patients from the Sharkia governorate Egypt. Hepat Mon 13(12):e12706. https://doi.org/10.5812/hepat mon. 12706

6. Ray SC, Arthur RR, Carella A, Bukh J, Thomas DL (2000) Genetic epidemiology of hepatitis $\mathrm{C}$ virus throughout Egypt. J Infect Dis 182(3):698-707

7. Pawlotsky J-M (2016) Hepatitis C virus resistance to direct-acting antiviral drugs in interferon-free regimens. Gastroenterology 151(1):70-86

8. Rajhi M, Ghedira K, Chouikha A, Djebbi A, Cheikh I, Yahia AB, Sadraoui A, Hammami W, Azouz M, Mami NB (2016) Phylogenetic analysis and epidemic history of hepatitis $\mathrm{C}$ virus genotype 2 in Tunisia North Africa. PLoS ONE 11(4):e0153761. https:// doi.org/10.1371/journal.pone.0153761

9. Abdel-Ghaffar TY, Sira MM, El Naghi S (2015) Hepatitis C genotype 4: the past, present, and future. World J Hepatol 7(28):2792-2810
10. Kliemann DA, Tovo CV, Da Veiga ABG, De Mattos AA, Wood C (2016) Polymorphisms and resistance mutations of hepatitis $\mathrm{C}$ virus on sequences in the European hepatitis $\mathrm{C}$ virus database. World J Gastroenterol 22(40):8910-8917

11. Pawlotsky J-M, Negro F, Aghemo A, Berenguer M, Dalgard O, Dusheiko G, Marra F, Puoti M, Wedemeyer H (2018) EASL recommendations on treatment of hepatitis C 2018. J Hepatol 69(2):461-511

12. Ahmed OA, Safwat E, Khalifa MO, Elshafie AI, Fouad MHA, Salama MM, Naguib GG, Eltabbakh MM, Sherief AF, AbdElsalam S (2018) Sofosbuvir plus daclatasvir in treatment of chronic hepatitis $\mathrm{C}$ genotype 4 infection in a Cohort of Egyptian patients: an experiment the size of Egyptian village. Int J Hepatol. https://doi.org/10.1155/2018/9616234

13. European Association for the Study of the Liver (2017) EASL recommendations on treatment of hepatitis C 2016. J Hepatol 66(1):153-194. https://doi.org/10.1016/j.jhep.2016.09.001

14. Pawlotsky JM (2014) New hepatitis C therapies: the toolbox, strategies, and challenges. Gastroenterology 146(5):1176-1192

15. Li Z, Liu Y, Zhang Y, Shao X, Luo Q, Guo X, Lin G, Cai Q, Zhao Z, Chong Y (2017) Naturally occurring resistanceassociated variants to hepatitis $\mathrm{C}$ virus direct-acting antiviral agents in treatment-naive $\mathrm{HCV}$ genotype 6a-infected patients. Biomed Res Int 2017: https://doi.org/10.1155/2017/9849823

16. Cai Q, Zhao Z, Liu Y, Shao X, Gao Z (2013) Comparison of three different $\mathrm{HCV}$ genotyping methods: core, NS5B sequence analysis and line probe assay. Int J Mol Med 31(2):347-352

17. Abdel-Hamid M, El-Daly M, Molnegren V, El-Kafrawy S, Abdel-Latif S, Esmat G, Strickland GT, Loffredo C, Albert J, Widell A (2007) Genetic diversity in hepatitis C virus in Egypt and possible association with hepatocellular carcinoma. J Gen Virol 88(5):1526-1531

18. Mellor J, Walsh E, Prescott L, Jarvis L, Davidson F, Yap P, Simmonds P (1996) Survey of type 6 group variants of hepatitis $\mathrm{C}$ virus in Southeast Asia by using a core-based genotyping assay. J Clin Microbiol 34(2):417-423

19. Bukh J, Miller RH, Purcell RH (1995) Genetic heterogeneity of hepatitis $\mathrm{C}$ virus: quasispecies and genotypes. Semin Liver Dis 15(1):41-63

20. Golemba MD, Culasso AC, Villamil FG, Bare P, Gadano A, Ridruejo E, Martinez A, Di Lello FA, Campos RH (2013) Hepatitis $\mathrm{C}$ virus diversification in Argentina: comparative analysis between the large city of Buenos Aires and the small rural town of O'Brien. PLoS ONE 8(12):e84007. https://doi.org/10.1371/ journal.pone.0084007

21. Tong Y-Q, Liu B, Liu H, Zheng H-Y, Gu J, Song E, Song C, Li Y (2015) Accurate genotyping of hepatitis C virus through nucleotide sequencing and identification of new HCV subtypes in China population. Clin Microbiol Infect 21(9):874.e9-874. e21

22. Elkady A, Tanaka Y, Kurbanov F, Sugauchi F, Sugiyama M, Khan A, Sayed D, Moustafa G, AbdEl-Hameed AR, Mizokami M (2009) Genetic variability of hepatitis C virus in South Egypt and its possible clinical implication. J Med Virol 81(6):1015-1023

23. Youssef A, Yano Y, Utsumi T, Abd El-alah EMM, Abd ElHameed AES, Serwah A-H, Hayashi Y (2009) Molecular epidemiological study of hepatitis viruses in Ismailia Egypt. Intervirology 52(3):123-131

24. Behrens S-E, Tomei L, De Francesco R (1996) Identification and properties of the RNA-dependent RNA polymerase of hepatitis C virus. EMBO J 15(1):12-22

25. Itakura J, Nagayama K, Enomoto N, Hamano K, Sakamoto N, Fanning LJ, Kenny-Walsh E, Shanahan F, Watanabe M (2005) Viral load change and sequential evolution of entire hepatitis $\mathrm{C}$ virus genome in Irish recipients of single source-contaminated anti-D immunoglobulin. J Viral Hepat 12(6):594-603 
26. Kanwal S, Mahmood T (2014) Occurrence of genetic modifications in core, 5' UTR and NS5b of HCV associated with viral response to treatment. Virol J 11(1):171

27. Lesburg CA, Cable MB, Ferrari E, Hong Z, Mannarino AF, Weber PC (1999) Crystal structure of the RNA-dependent RNA polymerase from hepatitis $C$ virus reveals a fully encircled active site. Nat Struct Mol Biol 6(10):937-943

28. Di Maio VC, Cento V, Mirabelli C, Artese A, Costa G, Alcaro S, Perno CF, Ceccherini-Silberstein F (2014) Hepatitis C virus genetic variability and the presence of NS5B resistance-associated mutations as natural polymorphisms in selected genotypes could affect the response to NS5B inhibitors. Antimicrob Agents Chemother 58(5):2781-2797

29. Waheed Y, Saeed U, Anjum S, Afzal MS, Ashraf M (2012) Development of global consensus sequence and analysis of highly conserved domains of the HCV NS5B protein. Hepat Mon 12(9):e6142. https://doi.org/10.5812/hepatmon.6142

30. Sesmero E, Thorpe IF (2015) Using the hepatitis $C$ virus RNA-dependent RNA polymerase as a model to understand viral polymerase structure, function and dynamics. Viruses 7(7):3974-3994

31. Qin W, Yamashita T, Shirota Y, Lin Y, Wei W, Murakami S (2001) Mutational analysis of the structure and functions of hepatitis C virus RNA-dependent RNA polymerase. Hepatology 33(3):728-737

32. Ogden KM, Ramanathan HN, Patton JT (2012) Mutational analysis of residues involved in nucleotide and divalent cation stabilization in the rotavirus RNA-dependent RNA polymerase catalytic pocket. Virology 431(1-2):12-20

33. Nikonov A, Juronen E, Ustav M (2008) Functional characterization of fingers subdomain-specific monoclonal antibodies inhibiting the hepatitis $\mathrm{C}$ virus RNA-dependent RNA polymerase. J Biol Chem 283(35):24089-24102

34. Ago H, Adachi T, Yoshida A, Yamamoto M, Habuka N, Yatsunami K, Miyano M (1999) Crystal structure of the RNAdependent RNA polymerase of hepatitis $\mathrm{C}$ virus. Structure 7(11):1417-1426

35. Zhong W, Uss AS, Ferrari E, Lau JYN, Hong Z (2000) De novo initiation of RNA synthesis by hepatitis $C$ virus nonstructural protein 5B polymerase. J Virol 74(4):2017-2022

36. Jaspe RC, Sulbarán YF, Sulbarán MZ, Loureiro CL, Rangel HR, Pujol FH (2012) Prevalence of amino acid mutations in hepatitis $\mathrm{C}$ virus core and NS5B regions among Venezuelan viral isolates and comparison with worldwide isolates. Virol J 9(214):1-7

37. Asahina Y, Izumi N, Enomoto N, Uchihara M, Kurosaki M, Onuki Y, Nishimura Y, Ueda K, Tsuchiya K, Nakanishi H (2005) Mutagenic effects of ribavirin and response to interferon/ ribavirin combination therapy in chronic hepatitis C. J Hepatol 43(4):623-629

38. Hamano K, Sakamoto N, Enomoto N, Izumi N, Asahina Y, Kurosaki M, Ueda E, Tanabe Y, Maekawa S, Itakura J (2005) Mutations in the NS5B region of the hepatitis C virus genome correlate with clinical outcomes of interferon-alpha plus ribavirin combination therapy. J Gastroenterol Hepatol 20(9):1401-1409

39. Legrand-Abravanel F, Nicot F, Izopet J (2010) New NS5B polymerase inhibitors for hepatitis C. Expert Opin Investig Drugs 19(8):963-975

40. Lam AM, Espiritu C, Bansal S, Steuer HMM, Niu C, Zennou V, Keilman M, Zhu Y, Lan S, Otto MJ (2012) Genotype and subtype profiling of PSI-7977 as a nucleotide inhibitor of hepatitis C virus. Antimicrob Agents Chemother 56(6):3359-3368. https ://doi.org/10.1128/AAC.00054-12

41. Ahmed A, Felmlee DJ (2015) Mechanisms of hepatitis C viral resistance to direct acting antivirals. Viruses 7(12):6716-6729

42. Donaldson EF, Harrington PR, O'Rear JJ, Naeger LK (2015) Clinical evidence and bioinformatics characterization of potential hepatitis $\mathrm{C}$ virus resistance pathways for sofosbuvir. Hepatology 61(1):56-65

43. Doss W, Shiha G, Hassany M, Soliman R, Fouad R, Khairy M, Samir W, Hammad R, Kersey K, Jiang D (2015) Sofosbuvir plus ribavirin for treating Egyptian patients with hepatitis $\mathrm{C}$ genotype 4. J Hepatol 63(3):581-585

44. Eltahla AA, Tay E, Douglas MW, White PA (2014) Cross-genotypic examination of hepatitis $\mathrm{C}$ virus polymerase inhibitors reveals a novel mechanism of action for thumb binders. Antimicrob Agents Chemother 58(12):7215-7224

45. Rigat KL, Lu H, Wang Y-K, Argyrou A, Fanslau C, Beno B, Wang Y, Marcinkeviciene J, Ding M, Gentles RG (2014) Mechanism of inhibition for BMS-791325, a novel non-nucleoside inhibitor of hepatitis C virus NS5B polymerase. J Biol Chem 289(48):33456-33468

46. Li Z, Chen Z-w, Li H, Ren H, Hu P (2017) Prevalence of hepatitis $\mathrm{C}$ virus-resistant association substitutions to direct-acting antiviral agents in treatment-naïve hepatitis $\mathrm{C}$ genotype $1 \mathrm{~b}$-infected patients in western China. Infect Drug Resist 10:377-392

47. Jiang M, Zhang EZ, Ardzinski A, Tigges A, Davis A, Sullivan JC, Nelson M, Spanks J, Dorrian J, Nicolas O (2014) Genotypic and phenotypic analyses of hepatitis $\mathrm{C}$ virus variants observed in clinical studies of VX-222, a nonnucleoside NS5B polymerase inhibitor. Antimicrob Agents Chemother 58(9):5456-5465

48. Poordad F, Lawitz E, Kowdley KV, Everson GT, Freilich B, Cohen D, Siggelkow S, Heckaman M, Menon R, Pilot-Matias T (2012) 12-week interferon-free regimen of ABT-450/R+ ABT$333+$ Ribavirin achieved svr 12 in more than $90 \%$ of treatmentnaive HCV genotype-1-infected subjects and $47 \%$ of previous non-responders. J Hepatol 56:S549-S550

49. Powdrill MH, Bernatchez JA, Götte M (2010) Inhibitors of the hepatitis $\mathrm{C}$ virus RNA-dependent RNA polymerase NS5B. Viruses 2(10):2169-2195

50. Howe AYM, Cheng H, Johann S, Mullen S, Chunduru SK, Young DC, Bard J, Chopra R, Krishnamurthy G, Mansour T (2008) Molecular mechanism of hepatitis $C$ virus replicon variants with reduced susceptibility to a benzofuran inhibitor, HCV796. Antimicrob Agents Chemother 52(9):3327-3338

51. Vermehren J, Sarrazin C (2012) The role of resistance in HCV treatment. Best Pract Res Clin Gastroenterol 26(4):487-503

52. Vega S, Neira JL, Marcuello C, Lostao A, Abian O, VelazquezCampoy A (2013) NS3 protease from hepatitis C virus: biophysical studies on an intrinsically disordered protein domain. Int $\mathbf{J}$ Mol Sci 14(7):13282-13306

53. Kwong A, Kim J, Lin C (2000) Structure and function of hepatitis $\mathrm{C}$ virus NS3 helicase. In: Hagedorn CH, Rice CM (eds) The Hepatitis C viruses. Springer, New York, pp 171-196

54. Grakoui A, McCourt D, Wychowski C, Feinstone S, Rice C (1993) Characterization of the hepatitis C virus-encoded serine proteinase: determination of proteinase-dependent polyprotein cleavage sites. J Virol 67(5):2832-2843

55. Kim J, Morgenstern K, Lin C, Fox T, Dwyer M, Landro J, Chambers S, Markland W, Lepre C, O'malley E, (1996) Crystal structure of the hepatitis $\mathrm{C}$ virus NS3 protease domain complexed with a synthetic NS4A cofactor peptide. Cell 87(2):343-355

56. Vallet S, Gouriou S, Nousbaum JB, Legrand-Quillien MC, Goudeau A, Picard B (2005) Genetic heterogeneity of the NS3 protease gene in hepatitis $C$ virus genotype 1 from untreated infected patients. J Med Virol 75(4):528-537

57. Tomei L, Failla C, Santolini E, De Francesco R, La Monica N (1993) NS3 is a serine protease required for processing of hepatitis C virus polyprotein. J Virol 67(7):4017-4026

58. Urbani A, Bazzo R, Nardi MC, Cicero DO, De Francesco R, Steinkühler C, Barbato G (1998) The metal binding site of the hepatitis C virus NS3 protease A spectroscopic investigation. J Biol Chem 273(30):18760-18769 
59. Failla C, Tomei L, De Francesco R (1995) An amino-terminal domain of the hepatitis C virus NS3 protease is essential for interaction with NS4A. J Virol 69(3):1769-1777

60. Heilek GM, Peterson MG (1997) A point mutation abolishes the helicase but not the nucleoside triphosphatase activity of hepatitis C virus NS3 protein. J Virol 71(8):6264-6266

61. Kim DW, Kim J, Gwack Y, Han JH, Choe J (1997) Mutational analysis of the hepatitis $\mathrm{C}$ virus RNA helicase. J Virol 71(12):9400-9409

62. Kim JW, Seo MY, Shelat A, Kim CS, Kwon TW, Lu H-h, Moustakas DT, Sun J, Han JH (2003) Structurally conserved amino acid W501 is required for RNA helicase activity but is not essential for DNA helicase activity of hepatitis C virus NS3 protein. J Virol 77(1):571-582

63. Lam AM, Keeney D, Frick DN (2003) Two novel conserved motifs in the hepatitis C virus NS3 protein critical for helicase action. J Biol Chem 278(45):44514-44524

64. Yao N, Hesson T, Cable M, Hong Z, Kwong A, Le H, Weber PC (1997) Structure of the hepatitis $C$ virus RNA helicase domain. Nat Struct Mol Biol 4(6):463

65. Mackintosh SG, Lu JZ, Jordan JB, Harrison MK, Sikora B, Sharma SD, Cameron CE, Raney KD, Sakon J (2006) Structural and biological identification of residues on the surface of NS3 helicase required for optimal replication of the hepatitis $\mathrm{C}$ virus. J Biol Chem 281(6):3528-3535

66. Lam AM, Frick DN (2006) Hepatitis C virus subgenomic replicon requires an active NS3 RNA helicase. J Virol 80(1):404-411

67. Tanji Y, Hijikata M, Satoh S, Kaneko T, Shimotohno K (1995) Hepatitis C virus-encoded nonstructural protein NS4A has versatile functions in viral protein processing. J Virol 69(3):1575-1581

68. Koch JO, Lohmann V, Herian U, Bartenschlager R (1996) In vitroStudies on the activation of the hepatitis $\mathrm{C}$ virus NS3 proteinase by the NS4A cofactor. Virology 221(1):54-66

69. Butkiewicz NJ, Wendel M, Zhang R, Jubin R, Pichardo J, Smith EB, Hart AM, Ingram R, Durkin J, Mui PW (1996) Enhancement of hepatitis $\mathrm{C}$ virus NS3 proteinase activity by association with NS4A-specific synthetic peptides: identification of sequence and critical residues of NS4A for the cofactor activity. Virology 225(2):328-338

70. Howe JA, Long J, Black S, Chase R, McMonagle P, Curry S, Thompson S, DiNubile MJ, Howe AY (2014) Clinical implications of detectable baseline hepatitis $\mathrm{C}$ virus-genotype 1 NS3/4A-protease variants on the efficacy of boceprevir combined with peginterferon/ribavirin. Open Forum Infect Dis. https://doi. org/10.1093/ofid/ofu078

71. Cento V, Landonio S, De Luca F, Di Maio VC, Micheli V, Mirabelli C, Niero F, Magni C, Rizzardini G, Perno CF (2013) Case report A boceprevir failure in a patient infected with $\mathrm{HCV}$ genotype $1 \mathrm{~g}$ : importance and limitations of virus genotyping prior to HCV protease-inhibitor-based therapy. Antivir Ther 18:645-648

72. Bae A, Sun S-C, Qi X, Chen X, Ku K, Worth A, Wong KA, Harris J, Miller MD, Mo H (2010) Susceptibility of treatmentnaive hepatitis $\mathrm{C}$ virus (HCV) clinical isolates to $\mathrm{HCV}$ protease inhibitors. Antimicrob Agents Chemother 54(12):5288-5297

73. Cento V, Mirabelli C, Salpini R, Dimonte S, Artese A, Costa G, Mercurio F, Svicher V, Parrotta L, Bertoli A (2012) HCV genotypes are differently prone to the development of resistance to linear and macrocyclic protease inhibitors. PLoS ONE 7(7):e39652. https://doi.org/10.1371/journal.pone.0039652

74. Fevery B, Verbinnen T, Peeters M, Janssen K, Witek J, Jessner W, De Meyer S, Lenz O (2017) Virology analyses of HCV genotype 4 isolates from patients treated with simeprevir and peginterferon/ribavirin in the Phase III RESTORE study. J Viral Hepat 24(1):28-36
75. Shepherd SJ, Abdelrahman T, MacLean AR, Thomson EC, Aitken C, Gunson RN (2015) Prevalence of HCV NS3 pre-treatment resistance associated amino acid variants within a Scottish cohort. J Clin Virol 65:50-53

76. Al-Salama ZT, Deeks ED (2017) Elbasvir/Grazoprevir: a review in chronic HCV genotypes 1 and 4. Drugs 77(8):911-921

77. Schnell G, Tripathi R, Beyer J, Reisch T, Krishnan P, Lu L, Dekhtyar T, Hall C, Vilchez RA, Pilot-Matias T (2015) Hepatitis $\mathrm{C}$ virus genotype 4 resistance and subtype demographic characterization of patients treated with ombitasvir plus paritaprevir/ ritonavir. Antimicrob Agents Chemother 59(11):6807-6815

78. Pilot-Matias T, Tripathi R, Cohen D, Gaultier I, Dekhtyar T, Lu L, Reisch T, Irvin M, Hopkins T, Pithawalla R (2015) In vitro and in vivo antiviral activity and resistance profile of the hepatitis $\mathrm{C}$ virus NS3/4A protease inhibitor ABT-450. Antimicrob Agents Chemother 59(2):988-997

79. Davies A, Singh KP, Shubber Z, Mills EJ, Cooke G, Ford N (2013) Treatment outcomes of treatment-naïve Hepatitis C patients co-infected with HIV: a systematic review and metaanalysis of observational cohorts. PLoS ONE 8(2):e55373. https ://doi.org/10.1371/journal.pone.0055373

80. Mohamed EK, Elbaz Tamer AELY, Gamal E (2016) Elbasvir and grazoprevir for chronic hepatitis $C$ genotypes 1 and 4. Expert Rev Clin Pharmacol 9(11):1413-1421

81. Asselah T, Kowdley KV, Zadeikis N, Wang S, Hassanein T, Horsmans Y, Colombo M, Calinas F, Aguilar H, de Ledinghen V (2018) Efficacy of glecaprevir/pibrentasvir for 8 or 12 weeks in patients with hepatitis $C$ virus genotype $2,4,5$, or 6 infection without cirrhosis. Clin Gastroenterol Hepatol 16(3):417-426

82. Shiha G, Esmat G, Hassany M, Soliman R, Elbasiony M, Fouad R, Elsharkawy A, Hammad R, Abdel-Razek W, Zakareya T (2018) Ledipasvir/sofosbuvir with or without ribavirin for 8 or 12 weeks for the treatment of $\mathrm{HCV}$ genotype 4 infection: results from a randomised phase III study in Egypt. Gut 68:721-728

83. Falade-Nwulia O, Suarez-Cuervo C, Nelson DR, Fried MW, Segal JB, Sulkowski MS (2017) Oral direct-acting agent therapy for hepatitis $\mathrm{C}$ virus infection: a systematic review. Ann Intern Med 166:637-648

84. Falade-Nwulia O, Sulkowski MS, Merkow A, Latkin C, Mehta SH (2018) Understanding and addressing hepatitis $C$ reinfection in the oral direct-acting antiviral era. J Viral Hepat 25:220-227

85. Mantovani LG, Cortesi PA, Strazzabosco M (2016) Effective but costly: How to tackle difficult trade-offs in evaluating health improving technologies in liver diseases. Hepatology 64(4):1331-1342

86. Bailey JR, Barnes E, Cox AL (2019) Approaches, progress, and challenges to hepatitis $\mathrm{C}$ vaccine development. Gastroenterology 156(2):418-430

87. Ward S, Lauer G, Isba R, Walker B, Klenerman P (2002) Cellular immune responses against hepatitis $\mathrm{C}$ virus: the evidence base 2002. Clin Exp Immunol 128:195-203

88. Barnes E, Folgori A, Capone S et al (2012) Novel adenovirusbased vaccines induce broad and sustained $\mathrm{T}$ cell responses to HCV in man. Sci Transl Med. https://doi.org/10.1126/scitranslm ed.3003155

89. Shoukry NH, Grakoui A, Houghton M, Chien DY, Ghrayeb J, Reimann KA, Walker CM (2003) Memory CD8+ T cells are required for protection from persistent hepatitis $\mathrm{C}$ virus infection. J Exp Med 197:1645-1655

90. Naeem A, Waheed Y (2017) Sequence analysis of hepatitis C virus nonstructural protein 3-4A serine protease and prediction of conserved B and T cell epitopes. Biomed Rep 7(6):563-566

91. Ikram A, Zaheer T, Awan FM, Obaid A, Naz A, Hanif R, Paracha RZ, Ali A, Naveed AK, Janjua HA (2018) Exploring NS3/4A, NS5A and NS5B proteins to design conserved subunit 
multi-epitope vaccine against $\mathrm{HCV}$ utilizing immunoinformatics approaches. Sci Rep 8(1):1-14

92. Polaris Observatory HCV Collaborators (2017) Global prevalence and genotype distribution of hepatitis $\mathrm{C}$ virus infection in 2015: a modelling study. Lancet Gastroenterol Hepatol 2:161-176

93. Reig M, Marino Z, Perello C, Inarrairaegui M, Ribeiro A, Lens S, Diaz A, Vilana R, Darnell A, Varela M, Sangro B, Calleja JL, Forns X, Bruix J (2016) Unexpected high rate of early tumor recurrence in patients with $\mathrm{HCV}$-related $\mathrm{HCC}$ undergoing interferon-free therapy. J Hepatol 65(4):719-726

94. Abdel-Hakeem MS, Shoukry NH (2014) Protective immunity against hepatitis C: many shades of gray. Front Immunol. https ://doi.org/10.3389/fimmu.2014.00274

95. Cerny A, McHutchison JG, Pasquinelli C, Brown ME, Brothers MA, Grabscheid B, Fowler P, Houghton M, Chisari FV (1995) Cytotoxic $\mathrm{T}$ lymphocyte response to hepatitis $\mathrm{C}$ virus-derived peptides containing the HLA A2.1 binding motif. J Clin Investig 95:521-530

96. Lechner F, Wong DK, Dunbar PR, Chapman R, Chung RT, Dohrenwend P, Robbins G, Phillips R, Klenerman P, Walker BD (2000) Analysis of successful immune responses in persons infected with hepatitis C virus. J Exp Med 191(9):1499-1512

97. Wong DK, Dudley DD, Dohrenwend PB, Lauer GM, Chung RT, Thomas DL, Walker BD (2001) Detection of diverse hepatitis $\mathrm{C}$ virus (HCV)-specific cytotoxic $\mathrm{T}$ lymphocytes in peripheral blood of infected persons by screening for responses to all translated proteins of HCV. J Virol 75:1229-1235

98. Smyk-Pearson S, Tester IA, Lezotte D, Sasaki AW, Lewinsohn DM, Rosen HR (2006) Differential antigenic hierarchy associated with spontaneous recovery from hepatitis $\mathrm{C}$ virus infection: implications for vaccine design. J Infect Dis 194(4):454-463

99. Bowen DG, Walker CM (2005) Mutational escape from CD8+ T cell immunity: HCV evolution, from chimpanzees to man. J Exp Med 201:1709-1714

100. Williams MA, Bevan MJ (2007) Effector and memory CTL differentiation. Ann Rev Immunol 25:171-192

101. Zhang Y, Liu Y, Moxley KM, Golden-Mason L, Hughes MG Jr, Liu T, Heemskerk MHM, Rosen HR, Nishimura MI (2010) Transduction of human T cells with a novel T-cell receptor confers anti-HCV reactivity. PLoS Pathog 6(7):e1001018. https:// doi.org/10.1371/journal.ppat.1001018

102. Ojiro K, Qu X, Cho H, Park J-J, Vuidepot A, Lissin N, Molloy PE, Bennett A, Jakobsen BK, Kaplan DE, Riley JL, Chang K-M (2017) Modulation of hepatitis C virus-specific CD8 effector T-cell function with antiviral effect in infectious hepatitis C virus coculture model. J Virol 91:e02129-e2216. https://doi. org/10.1128/JVI.02129-16

103. Zhang H, Zhang J, Chen L, Weng Z, Tian Y, Zhao H, Li Y, Chen L, Liang Z, Zheng H, Zhao W, Zhong S, Li Y (2017) Targeting naturally occurring epitope variants of hepatitis $C$ virus with high-affinity T-cell receptors. J Gen Virol 98(3):374-384

Publisher's Note Springer Nature remains neutral with regard to jurisdictional claims in published maps and institutional affiliations. 